Open Access

\title{
User scheduling for multicast transmission in high throughput satellite systems
}

\author{
Shuo Zhang, Min Jia, Yuming Wei and Qing Guo* (D)
}

*Correspondence: qguo@hit.edu.cn Harbin Institute of Technology, No.2 Yikuang Street, Harbin, China

\begin{abstract}
Adopting full frequency reuse in high throughput satellite (HTS) systems is expected to cope with huge communication demands and large user populations. Moreover, a kind of multicast transmission, which embeds the data of several users in each frame, can be employed to increase the efficiency of HTS systems. Multicast precoding is usually utilized in such systems to mitigate the co-frequency interference between beams and improve the efficiency of transmission. In this context, considering that the number of users may exceed the number of available communication resources in the system, we investigate user scheduling for the multicast transmission in HTS systems with full frequency reuse and multicast precoding. We perform user scheduling according to the user channel state information and decouple the scheduling problem into two phases: intra-beam and inter-beam scheduling. Intra-beam scheduling determines the users involved in the transmission of each frame with the purpose of reducing the influence of the multicast fashion. For intra-beam scheduling, we put forth a fixed-size user grouping algorithm. In contrast to previous studies, this algorithm takes the interference among beams into consideration during the scheduling. In inter-beam scheduling, user groups belonging to different beams are scheduled to improve the performance of the multiplexed transmission. An inter-beam scheduling algorithm is proposed to improve the fairness among users. The simulation results verify the superiority of the proposed algorithms in terms of fairness and spectral efficiency.
\end{abstract}

Keywords: HTS, Multicasting, User scheduling, Fairness

\section{Introduction}

The appearance of high throughput satellite (HTS) systems has brought new opportunities to satellite communication. In addition to the advantages of satellite communication, HTS systems have low communication cost and high throughput, which compensate the deficiencies of traditional satellite systems. Thanks to the rapid development of antenna technology [1-6], a large number of high-gain beams can be created and a high order of frequency reuse can be employed in the service area of the system to achieve this large throughput [7]. A four-color frequency reuse scheme is adopted in current HTS systems to avoid co-frequency interference between adjacent beams as well as obtaining

(C) The Author(s). 2020 Open Access This article is licensed under a Creative Commons Attribution 4.0 International License which permits use, sharing, adaptation, distribution and reproduction in any medium or format, as long as you give appropriate credit to the original author(s) and the source, provide a link to the Creative Commons licence, and indicate if changes were made. The images or other third party material in this article are included in the article's Creative Commons licence, unless indicated otherwise in a credit line to the material. If material is not included in the article's Creative Commons licence and your intended use is not permitted by statutory regulation or exceeds the permitted use, you will need to obtain permission directly from the copyright holder. To view a copy of this licence, visit http://creativecommons.org/licenses/by/4.0/. 
a fairly high frequency reuse factor [8]. As indicated in literature, present HTS systems have achieved a throughput over $300 \mathrm{Gbps}$ [9]. Partnering terrestrial communication systems, HTS is expected to address the challenges of huge traffic amounts and large user populations. Further, with the integration of satellite and terrestrial communication, HTS systems need to support various types of services [10].

Aggressive frequency reuse emerges in HTS systems to meet future communication demands [11]. Along with augmenting the available bandwidth in each beam, aggressive frequency reuse brings about the co-frequency interference between adjacent beams. This results in the interference-limited characteristics of the system. Considering the similarity between HTS communication and multiuser Multiple Input Multiple Output (MIMO) communication, precoding techniques can be employed to handle the co-frequency interference. With channel state information (CSI), precoding can bring throughput increase through the joint processing of the signals from different feeds [12]. Relevant studies show that the adoption of precoding in HTS systems with full frequency reuse can achieve performance improvement by comparison with ones that adopt four-color frequency reuse [13, 14].

Since coding schemes with long codewords are adopted in satellite communication to cope with the large fading loss of satellite links, satellite systems may statistically multiplex the data of different users belonging to the same beam in each frame to increase the encapsulation efficiency of the long frames [15]. Precisely, the data of a certain number of users are coded as a single codeword and the codeword is transmitted as a frame. Thus, each user can only obtain his data after successfully receiving the whole frame. Although this type of transmission can increase the efficiency of the system, it leads to a multicast fashion in each beam which complicates the precoding design [16]. Besides the precoding design, user scheduling according to the locations or CSIs of users becomes an emerging research field on account of the large user populations in such systems [12, 17]. A multicast-aware user scheduling algorithm was proposed to improve the performance of multicast transmission in HTS systems, and the simulation results indicated the throughput gains brought by user scheduling [18]. Since this study only investigated the multicast transmission with a fixed number of users, the algorithm might not apply to the situation where the number of users is much larger than the fixed number. For the user scheduling problem with a large number of users, the scheduling consists of two phases, i.e., intra-beam scheduling and inter-beam scheduling. Intra-beam scheduling, aiming at reducing the influence of the multicast fashion, is employed in each beam to constitute user groups, each of which consists of users sharing the same frame for transmission. Inter-beam scheduling determines the user groups (respectively belonging to different beams) that are involved in each multiplexed transmission among the beams. The two phases should be jointly handled to obtain the best performance, but most current studies only involve one of the two phases.

The multicast fashion in each beam limits the achievable rate of the users in each frame groups since users may experience different channel gains. In order to mitigate this impact, intra-beam scheduling aims at constituting user groups in which users have similar channel vectors [16]. Considering the invariable channel state of the users in fixed HTS communication, intra-beam scheduling was formulated as a clustering problem to guarantee that all the users can be served [19]. Clustering algorithms, such as spectral clustering [20] and K-means [19], were employed in intra-beam scheduling. These 
scheduling algorithms can obtain a fixed number of clusters with various cluster sizes. Fixed size user clustering, i.e., fixed size user grouping, has practical application for intrabeam scheduling for HTS systems because of the limited number of users in each frame. Indeed, standards with respect to satellite communication specify the length of the codewords of each coding scheme. For example, DVB-S2 standard adopts LDPC coding with fixed lengths of $16 \mathrm{kbit}$ and $64 \mathrm{kbit}$ [21]. The fixed length of the codeword in each frame suggests the necessity of fixed size clustering for intra-beam scheduling. A $k$-user grouping scheme was proposed to constitute fixed-size user groups for successive frame transmission by selecting the users based on the collinearity of channel vectors [16]. For the clustering scheduling problem, a fixed size clustering algorithm was proposed which randomly selected a user as the first user in each group and formed the group based on the similarity of the channel vectors between the first user and the others [22]. Further, the MaxDist algorithm, also a fixed size clustering algorithm, was proposed. The algorithm took a new approach to determine the first user in each group and outperformed the previous one that randomly selected each first user [19]. The aforementioned intrabeam scheduling algorithms can enhance the performance of the system. Nevertheless, these studies only focus on increasing the similarity of the channel vectors in each group through scheduling and have not taken the interference-limited characteristics of the system into consideration.

The inter-beam scheduling should maximize the orthogonality between the users in any two beams to improve the performance [23]. In contrast to general scheduling problems, each unit to be scheduled in inter-beam scheduling corresponds to a group of users instead of an individual user. Considering the complexity due to this fact, present HTS systems usually adopt random scheduling as the inter-beam scheduling scheme [24]. One of the few studies of inter-beam scheduling is a geographical scheduling algorithm, which partitioned the beams into zones and scheduled the users based on their locations [24]. This algorithm is appropriate for the situation where the users in HTS systems are uniformly distributed in each beam, but it does not account for the situation where some zones have no users. Besides user locations, user CSI can also be utilized in the scheduling. To our best knowledge, however, no previous studies have investigated the utilization of user CSI for inter-beam scheduling.

This paper investigates the user scheduling problem for multicast transmission in HTS systems with full frequency reuse. CSI of users is utilized for user scheduling. We divide the scheduling problem into intra-beam and inter-beam scheduling. For intrabeam scheduling, in view of the practicability of fixed size clustering, we pay attention to such a scheduling problem and seek for a new algorithm considering the existence of the co-frequency interference among the beams. For inter-beam scheduling, in addition to increasing the spectral efficiency of the system, the scheduling algorithm should ensure the fairness among users on account of the invariable channel state of users. One challenge associated with inter-beam scheduling is the handle of each unit to be scheduled, which corresponds to the CSIs of a number of users. The contributions of this paper can be concluded as follows:

- A fixed-size user grouping algorithm is proposed for intra-beam scheduling. In contrast to the previous studies, the interference-limited characteristics of the system is considered during the scheduling and a new similarity metric is utilized. 
- The concept of equivalent CSIs is introduced for inter-beam scheduling to simplify the inter-beam scheduling problem (making each scheduled unit only involve one CSI). Two forms of equivalent CSIs are presented.

- A scheduling algorithm according to equivalent CSIs is proposed for inter-beam scheduling aiming at improving the fairness among users.

The reminder of the paper is organized as follows. Section 2 models the multicast transmission of HTS systems with user scheduling, including the channel model, the signal model, and the precoding adopted in this paper. The problem statement and proposed algorithms for intra-beam and inter-beam scheduling are introduced in Sections 3 and 4, respectively. In Section 5, simulation results of different scheduling schemes, each including intra-beam and inter-beam scheduling, are presented in terms of average spectral efficiency and Jain's fairness index. Section 6 ends with the conclusion.

Notation: Boldface lowercase and uppercase letters denote vectors and matrices. $(\bullet)^{H}$ and $(\bullet)^{T}$ donate the conjugate transpose operator and the transpose operator. $\mathbb{C}$ and $\mathbb{R}$ donate the complex space and the real space. $\|\bullet\|$ correspond to the Frobenius norm. For a set, $|\bullet|$ donates the cardinality of the set, and for a vector, the result of $|\bullet|$ is the modulus vector of the vector. $[\bullet]_{m n}$ donates the $m, n$th element of the matrix. $C N\left(0, N_{0}\right), U[0,2 \pi)$ and $N\left(0, \sigma^{2}\right)$ donate the circularly symmetric Gaussian distribution, the uniform distribution, and the Gaussian distribution, respectively.

\section{System model and preliminaries}

We consider the downlink transmission (from the satellite to the user) of a geosynchronous orbit HTS that deploys full frequency reuse to provide services for fixed users in the satellite coverage area. The multibeam antenna on the satellite adopts the single feed per beam (SFB) structure and creates $N$ beams corresponding to $N$ feeds with the set $\mathcal{N}=\{1,2, \cdots, N\}$, as shown in Fig. 1 . In beam $n(n \in \mathcal{N}), M_{n}$ single-antenna users are distributed in the beam area with the user set $\mathcal{M}_{n}=\left\{1,2, \ldots, M_{n}\right\}$. The data of

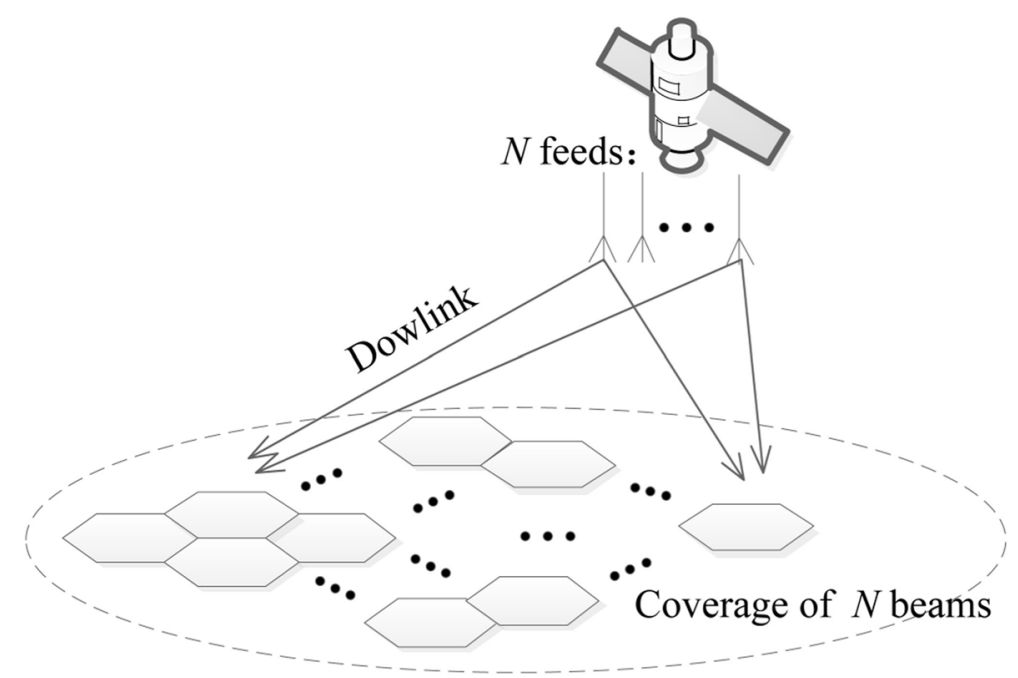

Fig. 1 The downlink transmission of the HTS system 
the users in each beam are embedded in frames. This leads to the multicast transmission. We assume that the transmission of frames in different beams are synchronized and the number of users involved in the transmission of each frame is lower than a fixed number $R$. Obviously, $R \ll\left|\mathcal{M}_{n}\right|$. User scheduling should sequentially determine the users participating in the transmission of each frame among all the users in these beams. In this paper, user scheduling is decoupled into intra-beam and inter-beam scheduling. The intra-beam scheduling, which is performed in each beam, divides the $M_{n}$ users into $K_{n}$ frame groups with the group set $\mathcal{K}_{n}=\left\{1,2, \ldots, K_{n}\right\}$ and the corresponding user set $\left\{\mathcal{U}_{1}^{(n)}, \mathcal{U}_{2}^{(n)}, \ldots, \mathcal{U}_{K_{n}}^{(n)}\right\}$ that satisfies $\sum_{k=1}^{K_{n}}\left|\mathcal{U}_{k}^{(n)}\right|=M_{n}$. Without loss of generality, we assume that the number of users in each beam is equal, i.e., $M_{n}=M, \forall n \in \mathcal{N}$. This assumption implies that each beam has the same number of frame groups, i.e., $K_{n}=K, \forall n \in \mathcal{N}$. The inter-beam scheduling partitions the frame groups of different beams into $K$ multiplexed groups $\left\{\mathcal{G}_{1}, \mathcal{G}_{2}, \ldots, \mathcal{G}_{K}\right\}$ with the group set $\mathcal{K}=\{1,2, \ldots, K\}$. Each multiplexed group $\mathcal{G}_{k}(k \in \mathcal{K})$ consists of $N$ frame groups, i.e., $\left|\mathcal{G}_{k}\right|=N, \forall k \in \mathcal{K}$, respectively, corresponding to frame group $l_{k, n} \in \mathcal{K}_{n}$ in beam $n$. After the precoding processing, the $N$ frames groups in a multiplexed group are simultaneously transmitted with the same frequency band. It takes $K$ successive frames to complete the transmission for all the users.

The diagram of multicast transmission with user scheduling for $N=2$ and $K=3$ is presented as an example in Fig. 2. The output of the intra-beam scheduling is the three frame groups in each beam. For each frame group, the data of the users are embedded in the same frame. Then, three multiplexed groups are obtained by the inter-beam scheduling. Each multiplexed group consists of two frames groups respectively from the two beams, and the data of the users in the two frames groups are transmitted with the same frequency band after precoding.

\subsection{Channel model}

We assume that the channel is flat-fading and the influence of inter-symbol interference is ignored. The channel coefficients between each user and the feeds can be expressed as a complex vector, and the channel vector of user $j \in \mathcal{M}_{n}$ in beam $n$ is indicated as $\mathbf{h}_{j}^{(n)} \in \mathbb{C}^{1 \times N}$. The $i$ th element stands for the channel coefficient between user $j$ and feed $i \in \mathcal{N}$, which can be expressed as

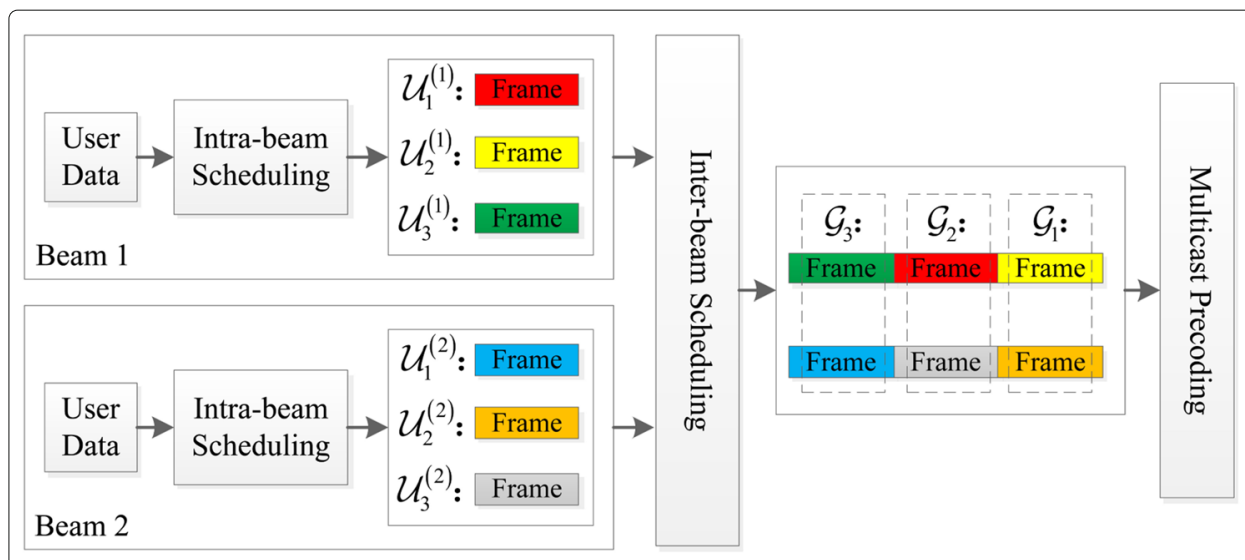

Fig. 2 Multicast transmission with user scheduling for $N=2$ and $K=3$ 


$$
h_{j, i}^{(n)}=G_{j, i}^{(n)} e^{j \varphi_{j, i}^{(n)}} .
$$

$G_{j, i}^{(n)}$ is the free space path loss of user $j$ from feed $i$, which can be expressed as [16]

$$
G_{j, i}^{(n)}=\frac{G_{R} b_{j, i}^{(n)}}{4 \pi \frac{d_{j, i}^{(n)}}{\lambda} \sqrt{K_{B} T_{R} B_{W}}}
$$

where $d_{j, i}^{(n)}$ is the distance between user $j$ and feed $i, \lambda$ is the wavelength, and $K_{B} T_{R} B_{W}$ is the noise power, in which $K_{B}, T_{R}$, and $B_{W}$ are the Boltzmann constant, the noise temperature, and the user link bandwidth, respectively. $G_{R}^{2}$ is the receiver antenna gain of the user, and $\left(b_{j, i}^{(n)}\right)^{2}$ is the multibeam antenna beam gain between user $j$ and feed $i$. For the SPF antenna, the beam gain can be approximated by [25]

$$
\left(b_{j, i}^{(n)}\right)^{2}=b^{2}\left(\theta_{j, i}^{(n)}\right)=G_{\max }\left(\frac{J_{1}(u)}{2 u}+36 \frac{J_{3}(u)}{u^{3}}\right)^{2},
$$

where $J_{1}$ and $J_{3}$ are respectively the first-kind Bessel function of order 1 and $3 . u=$ $2.07123 \sin \theta_{j, i}^{(n)} / \sin \theta_{3 d B}$, where $\theta_{3 d B}$ is the $3 \mathrm{~dB}$ angle of each beam and $\theta_{j, i}^{(n)}$ is the angle between the location of user $j$ and the $i$ th beam center.

The phase of the $i$ th channel coefficient of user $j$ is assumed as $[8,26]$

$$
\varphi_{j, i}^{(n)}=\theta_{j}^{(n)}+\delta_{i}
$$

where $\theta_{j}^{(n)} \sim U[0,2 \pi)$ is the same for the phases of all the channel coefficients of user $j$. It is the phase due to radio frequency (RF) signal propagation. Since the distance between a user and any feed is much longer than that between any two feeds, the phases caused by the RF signal propagation are almost the same for the same user. $\delta_{i} \sim N\left(0, \sigma^{2}\right)$ is the phase caused by the payload oscillator of feed $i$ [16]. $\delta_{i}$ is the same for the $i$ th channel coefficient of each user in the coverage area.

The phases of the channel vectors have a great effect on the system performance [27], and the phase variation $\delta_{i}$ cannot be obtained by channel estimation [28]. Thus, we make a reasonable assumption that the CSI available for the scheduling, i.e., available CSI, is donated as

$$
\hat{\mathbf{h}}_{j}^{(n)}=\left(\hat{h}_{j, 1}^{(n)}, \hat{h}_{j, 2}^{(n)}, \ldots, \hat{h}_{j, N}^{(n)}\right)=e^{j \theta_{j}^{(n)}} \cdot\left(G_{j, 1}^{(n)} G_{j, 2}^{(n)} \cdots G_{j, N}^{(n)}\right),
$$

which takes the imperfection estimation of phases into consideration.

\subsection{Signal model}

In the frame transmission of multiplexed group $k$, the channel vector of user $q$ in beam $n$ is donated as $\mathbf{h}_{q, l_{k, n}}^{(n)} \in \mathbb{C}^{1 \times N}\left(q \in \mathcal{U}_{l_{k, n}}^{(n)}, l_{k, n} \in \mathcal{K}_{n}\right)$, and the received signal of the user is written as

$$
y_{q, l_{k, n}}^{(n)}=\mathbf{h}_{q, l_{k, n}}^{(n)} \mathbf{P}_{k} \mathbf{s}_{k}+n_{q, l_{k, n}}^{(n)}
$$

where $n_{q, l_{k, n}}^{(n)} \sim C N\left(0, N_{0}\right)$ is the received noise and $\mathbf{s}_{k} \in \mathbb{C}^{N \times 1}$ is the transmitted signal from the $N$ feeds to the corresponding beams satisfying that $E\left[\left|s_{k, n}\right|^{2}\right]=1, \forall n \in \mathcal{N} . \mathbf{P}_{k} \in$ $\mathbb{R}^{N \times N}$ is the diagonal power factor matrix, and $\left[\operatorname{trace}\left(\mathbf{P}_{k} \mathbf{P}_{k}\right)\right]_{n n}$ is the transmitted power of feed $n$. Multicast precoding is adopted to reduce the interference between adjacent beams. For the signal $s_{k, n}$ of beam $n$, with the precoding vector $\mathbf{w}_{k, n} \in \mathbb{C}^{N}$, the received signal can be expressed as 


$$
y_{q, l_{k, n}}^{(n)}=\mathbf{h}_{q, l_{k, n}}^{(n)} \mathbf{w}_{k, n} s_{k, n}+\sum_{p \in \mathcal{N} \backslash\{n\}} \mathbf{h}_{q, l_{k, n}}^{(n)} \mathbf{w}_{k, p} s_{k, p}+n_{q, l_{k, n}}^{(n)} .
$$

The actual signal sent by the feeds is $\mathbf{x}_{k}=\sum_{n \in \mathcal{K}} s_{k, n} \mathbf{w}_{k, n}$. Assume that $\left\{s_{k, n}\right\}_{n=1}^{N}$ are mutually uncorrelated during the transmission. The transmitted power of all the feeds is $\sum_{n \in \mathcal{N}} \mathbf{w}_{k, n}^{H} \mathbf{w}_{k, n}$, and the transmitted power of feed $n$ is $\left(\sum_{n^{\prime} \in \mathcal{N}} \mathbf{w}_{k, n^{\prime}} \mathbf{w}_{k, n^{\prime}}^{H}\right)_{n n}$.

The signal to interference plus noise ratio (SINR) of the user after precoding is

$$
\operatorname{SINR}_{q, l_{k, n}}^{(n)}=\frac{\left|\mathbf{h}_{q, l_{k, n}}^{(n)} \mathbf{w}_{k, n}\right|^{2}}{\sum_{p \in \mathcal{N} \backslash\{n\}}\left|\mathbf{h}_{q, l_{k, n}}^{(n)} \mathbf{w}_{k, p}\right|^{2}+N_{0}} .
$$

The actual data rate of the users in each frame group depends on the lowest SINR of the frame group members because of the multicast fashion. Thus, based on Shannon formulation, the spectral efficiency of frame group $l_{k, n}$ in beam $n$ can be expressed as

$$
C_{l_{k, n}}^{(n)}=\log _{2}\left(1+\min _{q \in \mathcal{U}_{l_{k, n}}^{(n)}} \operatorname{SINR}_{q, l_{k, n}}^{(n)}\right) .
$$

For multiplexed group $k$ which consists of frame groups $\left\{\mathcal{U}_{l_{k, n}}^{(n)}, \forall n \in \mathcal{N}\right\}$, the average spectral efficiency per beam can be expressed as

$$
C_{k}=\frac{\sum_{n \in \mathcal{N}} C_{l_{k, n}}^{(n)}}{N}
$$

\subsection{Multicast precoding}

The precoding design influences the system performance. For multicast transmission, the precoding problem is NP-hard for optimization objectives such as minimizing the transmitted power, maximizing the fairness among users [29, 30]. These algorithms are complex and involve iterative interior point methods during the calculation of precoding vectors. A low-complex precoding algorithm, which was a one-shot design, was proposed to improve the transmission efficiency by limiting the interference between adjacent beams in HTS systems [16]. The aforementioned algorithms, however, all have higher complexity than the average minimum mean squared error (MMSE) scheme, which was first proposed for multicast transmission in HTS systems [31]. Considering that this scheme can achieve satisfactory performance with low complexity, we adopt it as the multicast precoding scheme in this paper. As the available CSI shown in (5) has the same phase for each feed link, we leave out the phases of the channel coefficients. Thus, in multiplexed group $k$, the average available CSI of frame group $l_{k, n}$ used for multicast precoding is

$$
\hat{\mathbf{h}}_{l_{k, n}}^{(n)}=\frac{\sum_{q \in \mathcal{U}_{l_{k, n}^{(n)}}}\left|\hat{\mathbf{h}}_{q, l_{k, n}}^{(n)}\right|}{\left|\mathcal{U}_{l_{k, n}}^{(n)}\right|} .
$$

The channel matrix used for precoding is $\mathbf{H}_{k}=\left(\hat{\mathbf{h}}_{l_{k, 1}}^{(1)^{T}}, \hat{\mathbf{h}}_{l_{k, 2}}^{(2)}, \ldots, \hat{\mathbf{h}}_{l_{k, N}}^{(N)}\right)^{T}$. For the sum transmitted power $P_{T}$, the precoding matrix $\mathbf{W}_{k}=\left(\mathbf{w}_{k, 1}, \mathbf{w}_{k, 2}, \ldots, \mathbf{w}_{k, N}\right)$ is

$$
\mathbf{W}_{k}=\gamma \mathbf{H}_{k}^{H}\left(\mathbf{H}_{k} \mathbf{H}_{k}^{H}+\frac{N}{P_{T}}\right)^{-1}
$$


where $\gamma$ is the power factor, fulfilling trace $\left(\mathbf{W}_{k} \mathbf{W}_{k}^{H}\right) \leq P_{T}$ for the sum power constraint or $\left[\mathbf{W}_{k} \mathbf{W}_{k}^{H}\right]_{n n} \leq P_{T} / N, \forall n \in \mathcal{N}$ for per-antenna power constraints.

\section{Intra-beam scheduling}

In this section, the intra-beam scheduling problem is first introduced and a fixed-size user grouping algorithm, named Mod-MaxDist, is proposed.

\subsection{Problem statement}

Performed respectively in each beam, the intra-beam scheduling divides the users into frame groups according to the CSIs. The channel condition differences in a frame group influence the data rate of the multicast transmission. An intra-beam scheduling algorithm should constitute frame groups with users having similar channel conditions to enhance the efficiency of the transmission. The date rate of the users in each frame group depends on the lowest SINR of the members. According to (8), not only the similarity of the channel vectors of the users in each frame group, but also the interference from the other beams has effects on the performance of the multicast transmission.

During the algorithm design, a key issue is to select a proper metric to measure the similarity between the user channel vectors to facilitate the scheduling. For clustering scheduling algorithms involving an iterative process, since similarity metrics need to satisfy the conditions of identity of indiscernibles, symmetry, and triangle inequality [32], the Euclidean distance between the user locations or channel vectors is often utilized as a similarity metric. However, for scheduling algorithms without an iterative process, more appropriate metrics can be utilized regardless of these conditions.

In this paper, the objective of the intra-beam scheduling is to design a fixed-size user grouping algorithm that allocates $\left|\mathcal{M}_{n}\right|$ users into $K_{n}$ frame groups $\left\{\mathcal{U}_{1}^{(n)}, \mathcal{U}_{2}^{(n)}, \ldots, \mathcal{U}_{K_{n}}^{(n)}\right\}$ according to the available CSIs. Specifically, the algorithm should utilize a proper metric to measure the similarity of the channel conditions and take the interference-limited characteristics of the system into account.

\subsection{Mod-MaxDist}

The Mod-MaxDist algorithm sequentially establishes the fixed-size frames groups. For each frame group, the first user is selected employing the approach of MaxDist [19], i.e., selecting the outlier among the users as the first user, then making a certain number of users whose channel vectors have higher similarity to the channel vector of the first user than the others as the frame group members. The highlights of the proposed algorithm, also the main differences from MaxDist, lie in:

- The CSI used for scheduling is redefined based on the available CSI considering the interference-limited characteristics of the system. For a user in a beam, the redefined CSI only relates to the channel coefficients of the feed links that cause high interference to the beam. For beam $n$, the set of feed links that cause high interference is donated as $\mathcal{N}_{n}$. Notice that $\hat{h}_{j, n}^{(n)}$ is not considered during the scheduling, i.e., $n \notin \mathcal{N}_{n}$. The determination of $\mathcal{N}_{n}$ depends on the beam pattern of the HTS. For example, $\mathcal{N}_{n}$ can be defined as a set that comprises the six adjacent beams surrounding beam $n$. For user $j$ in beam $n$, the refined CSI $\tilde{\mathbf{h}}_{j}^{(n)}=\left(\tilde{h}_{j, 1}^{(n)}, \tilde{h}_{j, 2}^{(n)}, \ldots, \tilde{h}_{j, N}^{(n)}\right)$ satisfies 


$$
\tilde{h}_{j, i}^{(n)}=\left\{\begin{array}{ll}
\hat{h}_{j, i}^{(n)} & i \in \mathcal{N}_{n} \\
0 & i \notin \mathcal{N}_{n}
\end{array} .\right.
$$

The CSI set of beam $n$ can be denoted as $\tilde{\mathcal{H}}^{(n)}=\left\{\tilde{\mathbf{h}}_{1}^{(n)}, \tilde{\mathbf{h}}_{2}^{(n)}, \ldots, \tilde{\mathbf{h}}_{M_{n}}^{(n)}\right\}$. The redefinition of the CSI reduces the complexity of the intra-beam scheduling. Moreover, the algorithm has good scalability since only a fixed part of the CSI is necessary.

- A new similarity metric is utilized for the scheduling algorithm. Existing algorithms usually utilize the Euclidean distance of channel coefficients or user locations as a similarity metric to achieve clustering. Since no iterative process is involved in the fixed size clustering, the three conditions related to the metric no longer need to be satisfied. Thus, more effective metrics can be utilized. For a selected outlier (the first user in a frame group) and user $j$ with the corresponding redefined CSIs $\mathbf{g}$ and $\tilde{\mathbf{h}}_{j}^{(n)}$, the metric, i.e., the norm of the inner product (NoIP), is defined as

$$
P_{k j}^{(n)}=\left|\mathbf{g} \tilde{h}_{j}^{(n)^{H}}\right| .
$$

The geometric meaning of NoIP is the projection from $\tilde{\mathbf{h}}_{j}^{(n)}$ to $\mathbf{g}$. This metric has lower computational complexity than the Euclidean distance, and its expression is similar to the desired signal, i.e., the molecular part of the SINR.

- The situation where $M_{n}$ is a non-integer multiple of $R$ is considered in the algorithm. In this situation, the size of one of the frame groups is smaller than $R$. Our proposed algorithm sets the first frame group (containing the first outlier) as the frame group with the smaller group size.

The details of the algorithm are shown in Algorithm 1. $r_{k_{n}}$ stands for the size of frame group $k_{n}$, i.e., the number of elements in $\mathcal{U}_{k_{n}}^{(n)}$. Algorithm 1 needs to be ran $N$ times to complete the intra-beam scheduling process of all the beams. The number of frame groups $K_{n}$ and the frame group size are first determined based on the values of $R$ and $M_{n}$. The $M_{n}$ users are allocated to the frame groups by calculating and comparing the NoIPs

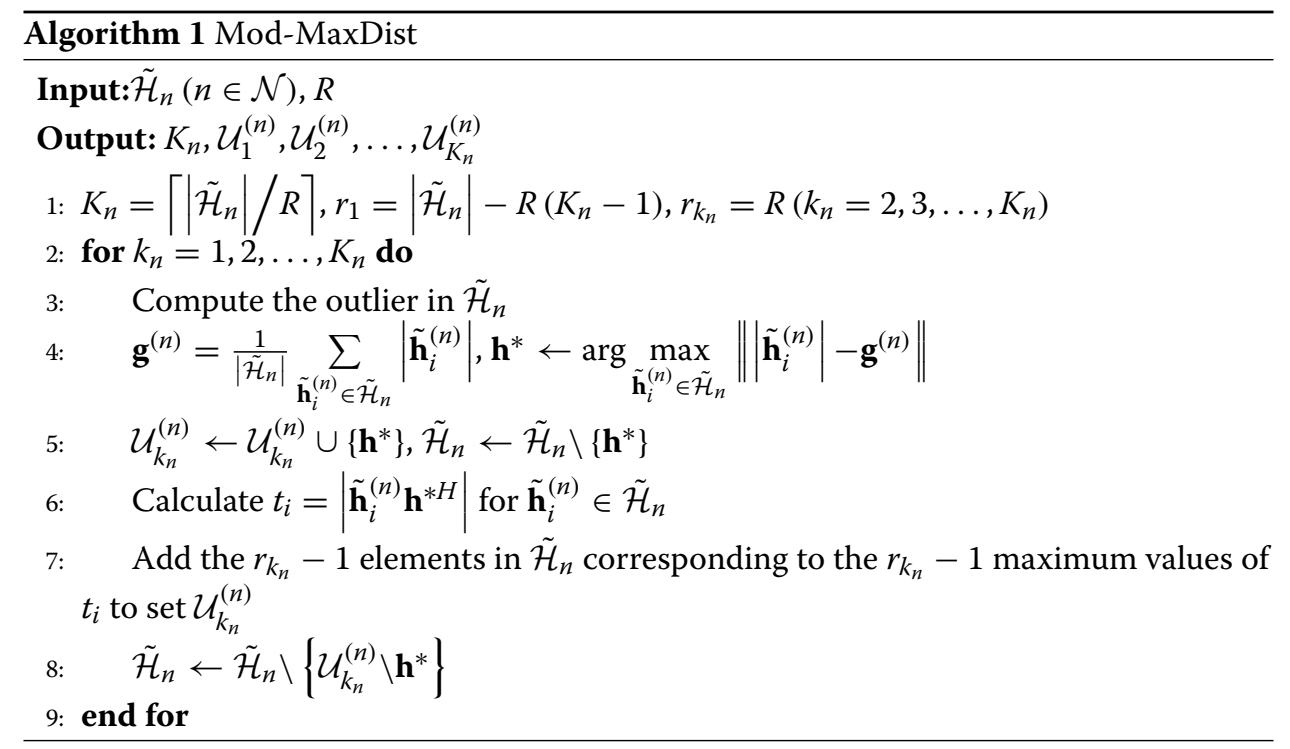


between the users and the first user in each frame group until all the $K_{n}$ frame groups are formed.

\section{Inter-beam scheduling}

The inter-beam scheduling partitions the frame groups obtained from the intra-beam scheduling into multiplexed groups with the aim of enhancing the performance of multiplexed transmission. As analyzed before, the main challenge of the inter-beam scheduling is that each scheduled unit corresponds to a set of available CSIs. We handle this by introducing a concept of equivalent CSIs to make each unit correspond to only one CSI. Thus, the inter-beam scheduling contains two steps:

- Calculation of equivalent CSIs: Calculate the equivalent CSI of each frame group based on the available CSIs of the users in the frame group. Two forms of equivalent CSIs are presented.

- Frame scheduling: According to equivalent CSIs, frame groups are divided into multiplexed groups based on the relationships among the frame groups. A heuristic scheduling algorithm, named fairness scheduling, is put forth.

The details of the two steps are presented in the following part.

\subsection{Equivalent CSI}

An equivalent CSI is a representative of a frame group during the scheduling. It should be determined considering the available CSIs of the frame group members. For the multicast transmission in HTS systems, we propose two forms of equivalent CSIs. For beam $n$, the set of equivalent CSIs used for the intra-beam scheduling is denoted as $\mathcal{C}^{(n)}=\left\{\mathbf{c}_{1}^{(n)}, \mathbf{c}_{2}^{(n)}, \ldots, \mathbf{c}_{K}^{(n)}\right\}$ in this paper.

\subsubsection{The direction vector of the average available CSI}

The direction vector of the average available CSI can serve as the equivalent CSI of a frame group. For frame group $k_{n} \in \mathcal{K}_{n}$, the direction vector can be expressed with Eq. (11), i.e., $\mathbf{c}_{k_{n}}^{(n)}=\hat{\mathbf{h}}_{k_{n}}^{(n)} /\left\|\hat{\mathbf{h}}_{k_{n}}^{(n)}\right\|$. This form implies the available CSIs for all the frame group members. Considering that average available CSIs are the inputs of the precoding, this form of equivalent CSIs is appropriate for average MMSE precoding.

\subsubsection{The center of the frame group}

The center of the available CSIs corresponding to the frame group can serve as the equivalent CSI. To obtain the center $\mathbf{c}_{k_{n}}^{(n)} \in \mathcal{K}_{n}$, we need to solve the following optimization problem for each frame group:

$$
\begin{array}{r}
\max _{\mathbf{c}_{k_{n}}^{(n)}} \min _{q \in \mathcal{U}_{k_{n}}^{(n)}} \frac{\left|\mathbf{c}_{k_{n}}^{(n)} \hat{\mathbf{h}}_{q, k_{n}}^{(n)}\right|}{\left\|\hat{\mathbf{h}}_{q, k_{n}}^{(n)}\right\|} \\
\text { s.t }\left\|\mathbf{c}_{k_{n}}^{(n)}\right\|=1
\end{array}
$$

Indeed, problem (15) is equivalent to the single-group multicast precoding problem, which is NP-hard [33]. The calculation of the center has high computational complexity. This form of equivalent CSIs can reflect the feature of the frame group especially for large frame group sizes. 


\subsection{Frame scheduling}

The adoption of equivalent CSIs simplifies the inter-beam scheduling. The fact that users are associated with specific beams results in constraints on the scheduling. The following part presents the problem statement and the proposed fairness scheduling algorithm for the inter-beam scheduling.

\subsubsection{Problem statement}

Frame scheduling forms $K$ multiplexed groups $\left\{\mathcal{G}_{1}, \mathcal{G}_{2}, \ldots, \mathcal{G}_{K}\right\}$ according to the equivalent CSI sets $\left\{\mathcal{C}^{(1)}, \mathcal{C}^{(2)}, \ldots, \mathcal{C}^{(N)}\right\}$. Thanks to the adoption of equivalent CSIs, the frame scheduling can learn from the multiuser MIMO scheduling. Thus, the frame scheduling can improve the performance of the multiplexed transmission with multicast precoding by ensuring that any two equivalent CSIs of the frame groups in the same multiplexed group have small similarity as far as possible.

As introduced before, the objective of the inter-beam scheduling is to maximize the fairness among the users in all the frame groups. That is to say, the frame scheduling should consider the performance of not only the scheduled frame groups but also the unscheduled ones. Thus, the design objective can be expressed as

$$
\max _{\mathcal{G}_{1}, \mathcal{G}_{2}, \ldots, \mathcal{G}_{K}} \min _{k \in \mathcal{K}} C_{k}
$$

where $C_{k}$ is the average spectral efficiency per beam for $\mathcal{G}_{k}$ and is calculated with (10). The constraints that should be satisfied during the scheduling are:

- $\forall k \in \mathcal{K}$, the $N$ elements in $\mathcal{G}_{k}$ respectively belong to $\mathcal{K}_{1}, \mathcal{K}_{2}, \ldots \mathcal{K}_{N}\left(\mathcal{K}_{n}\right.$ corresponding to the elements in $\mathcal{C}^{(n)}$ );

- $\forall k \in \mathcal{K},\left|\mathcal{G}_{k}\right|=N$.

The number of available scheduling results is $(K !)^{N}$, which makes it impractical to perform the exhaustive search. We focus on heuristic algorithms with low complexity. Aiming at maximizing the fairness, the proposed scheduling algorithm should take the relationships between the frame groups of any two beams into consideration during the scheduling.

\subsubsection{The fairness scheduling algorithm}

A heuristic algorithm, named the fairness scheduling (FS) algorithm, is proposed to deal with the frame scheduling problem raised above. The algorithm sequentially allocates the $K$ frame groups in each beam $\mathcal{K}_{n}(n \in \mathcal{N})$ to the $K$ multiplexed groups $\left\{\mathcal{G}_{1}, \mathcal{G}_{2}, \ldots, \mathcal{G}_{K}\right\}$ based on $\mathcal{C}^{(n)}(n \in \mathcal{N})$ until $\left|\mathcal{G}_{k}\right|=N, \forall k \in \mathcal{K}$. The details are shown in Algorithm 2. To improve the fairness among the users in all the beams, the beams are scheduled in a certain order based on the amount of interference that each beam suffers and the beam pattern. Precisely, the beam suffering more interference should be first scheduled to avoid poor scheduling results. For the scheduling of the frame groups in the $n$th beam, a pairing algorithm makes a one-to-one mapping between the $K$ frame groups in the $n$th beam and the $K$ multiplexed group sets $\left\{\mathcal{G}_{1}, \mathcal{G}_{2}, \ldots, \mathcal{G}_{K}\right\}$ based on a relation matrix $\mathbf{Q} \in \mathbb{R}^{K \times K}$. $\mathbf{Q}$ represents the correlation between the $K$ elements in $\mathcal{C}^{n}$ and the $K$ incomplete multiplexed groups.

The following part introduces the calculation of the relation matrix $\mathbf{Q}$ and our proposed pairing algorithm. 


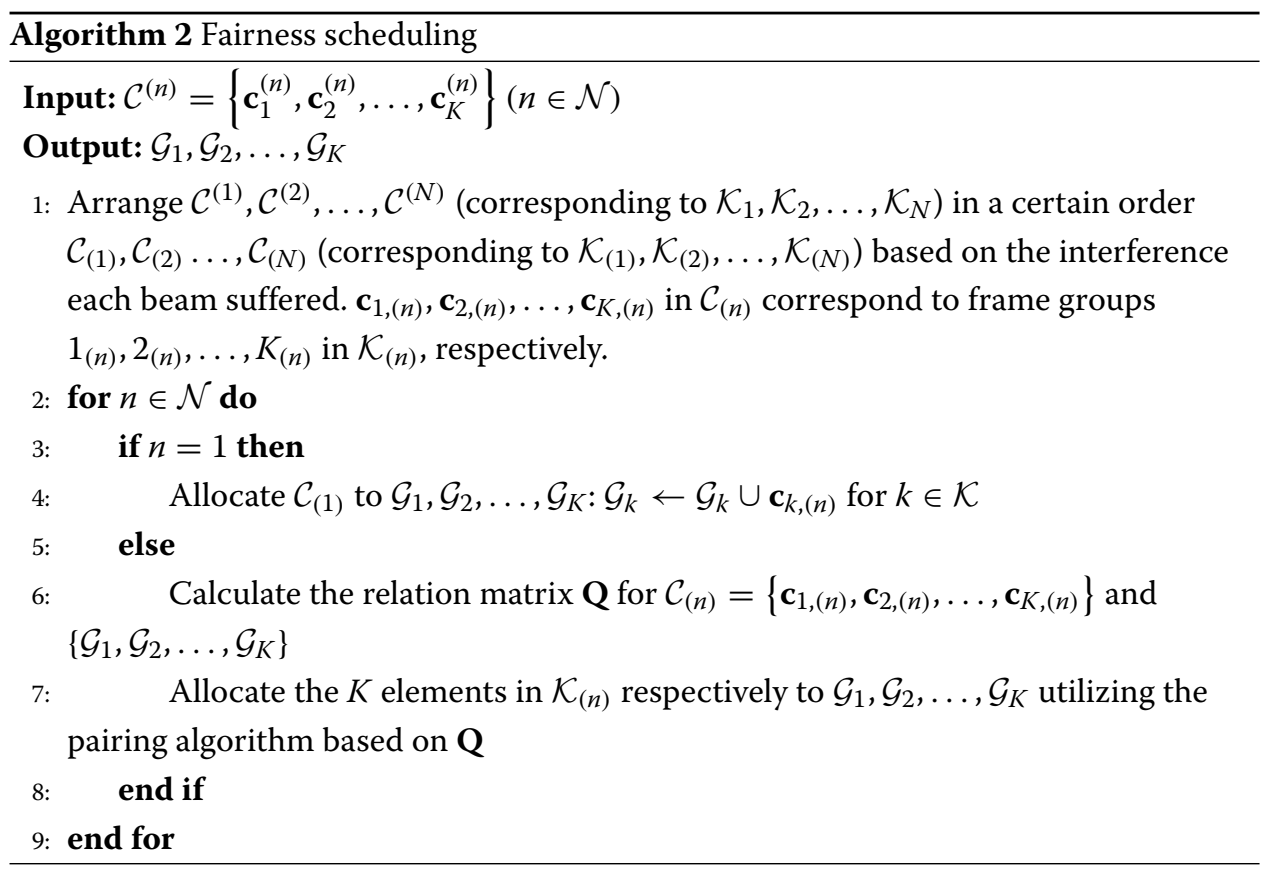

Calculation of the relation matrix The $i, j \operatorname{th}(i, j \in \mathcal{K})$ element of the relation matrix $\mathbf{Q}$ stands for the projection of the equivalent CSI of the $j$ th frame group in the beam to be scheduled on the space spanned by the equivalent CSIs of the frame groups in the $i$ th incomplete multiplexed group. For the $n$th beam to be scheduled, the equivalent CSI of the $j$ th frame group is $\mathbf{c}_{j,(n)}$. For the group set $\mathcal{G}_{i}(i \in \mathcal{K})$, the equivalent CSIs of the frame groups can constitute a matrix $\mathbf{H}_{\mathcal{G}, i} \in \mathbb{C}^{\left|\mathcal{G}_{i}\right| \times N}$, of which each row is an equivalent CSI. Obviously, the incomplete multiplexed groups satisfy that $\left|\mathcal{G}_{i}\right|=n-1, \forall i \in \mathcal{K} .[\mathbf{Q}]_{i, j}$, the projection, can be expressed as

$$
[\mathbf{Q}]_{i, j}=\left|\mathbf{c}_{j,(n)} \mathbf{H}_{\mathcal{G}, i}^{H}\left(\mathbf{H}_{\mathcal{G}, i} \mathbf{H}_{\mathcal{G}, i}^{H}\right)^{-1} \mathbf{H}_{\mathcal{G}, i}\right|^{2} .
$$

A large value of $[\mathbf{Q}]_{i, j}$ means that the $j$ th frame group has low similarity to the multiplexed group $\mathcal{G}_{i}$, and the interference may be strong if this frame group is allocated to $\mathcal{G}_{i}$.

The pairing algorithm The pairing algorithm selects $K$ elements $q_{1}, q_{2}, \ldots, q_{K}$ from $\mathbf{Q}$, fulfilling that not only the row numbers but also the column numbers of these elements are mutually unequal. $\left\{q_{k}\right\}_{k=1}^{K}$ represent the one-to-one mappings between the $K$ frame groups of the beam to be scheduled and the multiplexed group sets $\mathcal{G}_{1}, \mathcal{G}_{2}, \ldots, \mathcal{G}_{K}$. Specifically, $q_{k}=[\mathbf{Q}]_{w, v}$ means that the $v$ th frame group is scheduled to multiplexed group $\mathcal{G}_{w}$ in the $k$ th one-to-one mapping.

Aiming at maximizing the fairness during the scheduling, the pairing algorithm has the design purpose:

$$
\min _{\mathcal{C}_{(n)} \rightarrow\left\{\mathcal{G}_{1}, \mathcal{G}_{2}, \ldots, \mathcal{G}_{K}\right\}} \max _{k \in \mathcal{K}} q_{k} .
$$

The number of available pairing results is $K$ ! as there are $K^{2}$ elements in $\mathbf{Q}$. When $K$ is small, the optimal pairing results are easy to achieve, but the complexity of pairing increases with the rise in $K$. The pairing algorithm belongs to heuristic selection 
algorithms and the details of it are shown in Algorithm 3. The selection of the elements in the matrix is carried out by comparing the value of $[\mathbf{Q}]_{i, j}$ with a threshold $\alpha$. In this way, the search space is reduced. For the selection of the $K$ elements, only pairing results that satisfy $\forall k \in \mathcal{K}, q_{k} \leq \alpha$ are accepted; otherwise, the threshold is raised and a new selection starts until accepted results are achieved. The initial value of $\alpha$ is

$$
\alpha=\max \left\{\operatorname{maxmin}_{i \in \mathcal{K}}[\mathbf{Q}]_{i, j} \operatorname{maxmin}_{j \in \mathcal{K}}[\mathbf{Q}]_{i, j}\right\} .
$$

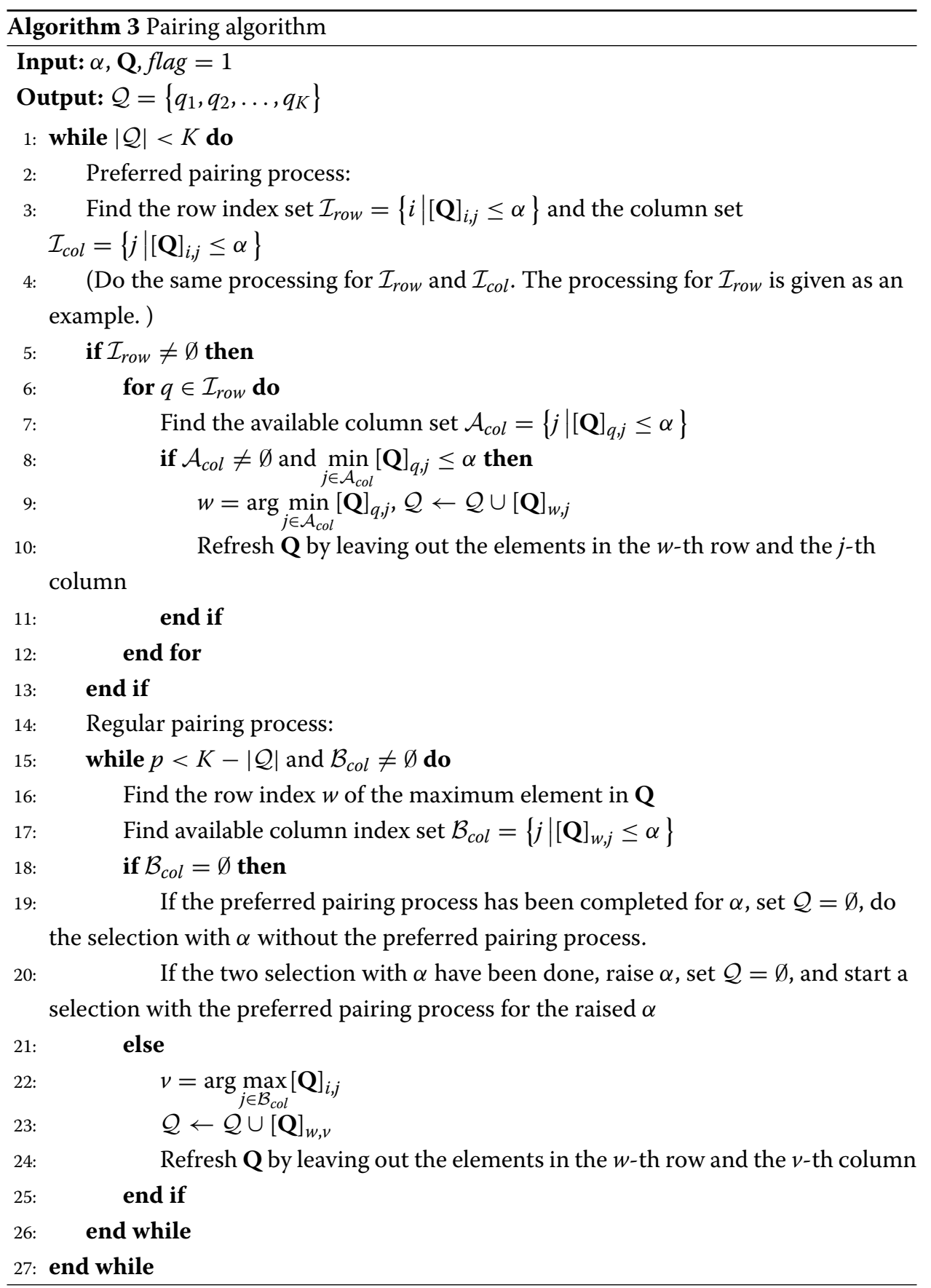


Two selections are performed for each $\alpha$. The first selection includes a preferred pairing process (lines 3 to 13) to achieve good pairing results as far as possible. Through the process, some frame groups and multiplexed groups are scheduled preferentially. If no available results are obtained after the first selection, the selection with the threshold $\alpha$ restarts without the preferred pairing process.

No backtracking processing is involved in the pairing algorithm. Remarkably, the proposed pairing algorithm can be extended to other pairing situations where a relation matrix is available.

\section{Simulation result}

A seven-beam scenario $(N=7)$, where a beam is surrounded by six beams, is considered in the simulation. Full frequency reuse is adopted among the seven contiguous beams, and one frequency band is involved in the data transmission of the beams. $M$ users are randomly distributed in each beam. The simulation parameters are $f=20 \mathrm{GHz}$, $B_{W}=41.7 \mathrm{MHz}, K_{B}=1.38 \times 10^{-23} \mathrm{~K} / \mathrm{J}, G_{\max }=52 \mathrm{~dB}, G_{R}^{2}=41.7 \mathrm{~dB}, T_{R}=207 \mathrm{~K}$, $\theta_{3 d B}=0.2^{\circ}$, and $\sigma=2^{\circ}$ for phase variation $[16,34]$. The height of the GEO satellite is $35786 \mathrm{~km}$. For different values of the user number $M$, the multiplexed group number $K$, the transmitted power $P_{T}$, and the maximum frame group size $R$, the simulation results are obtained over $N_{m c}$ Monte Carlo runs. For Algorithm 3, $\alpha$ is raised by 0.1 each time in the simulation.

The direction vector of the average available CSI is used as the equivalent CSI for the inter-beam scheduling. The sum power constraint is considered for the average MMSE precoding. Two intra-beam scheduling algorithms, i.e., MaxDist [19] (indicated by MD) and the proposed Mod-MaxDist (indicated by Mod-MD) and two inter-beam scheduling algorithms, i.e., random scheduling (indicated by RS) and our fairness scheduling (indicated by FS) proposed in Section 4, are involved in the simulation. Thus, the performances

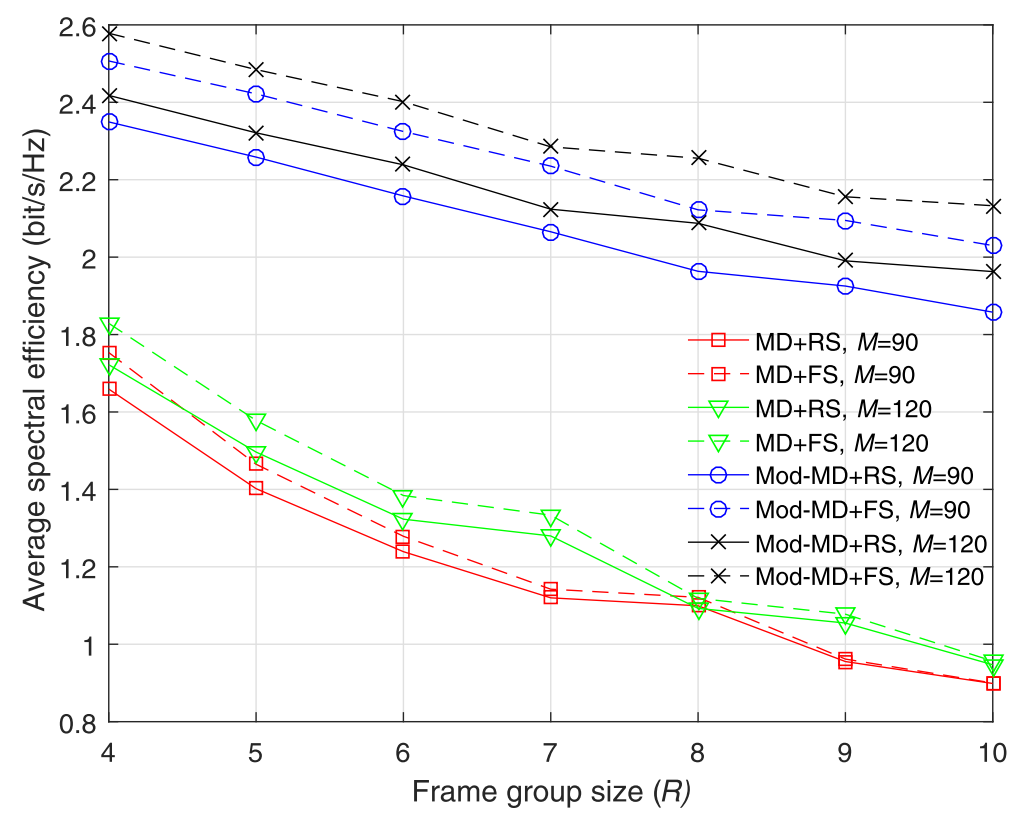

Fig. 3 Average spectral efficiency verse $R, P_{T}=10 \mathrm{~dB}$ 
of the four scheduling schemes, i.e., $M D+R S, M D+F S$, Mod-MD+RS, and Mod-MD+FS, are compared with each other.

\subsection{Average spectral efficiency}

The average spectral efficiency is calculated with (10). Figure 3 depicts the average spectral efficiency verse $R$ under different scheduling schemes with $M=90,120$. It is observed that the average spectral efficiency declines with the growth in $R$. This is excepted as larger $R$ may reduce the spectral efficiency of the multicast transmission. It is observed that Mod-MD significantly outperforms MD, and the scheduling schemes with FS have better

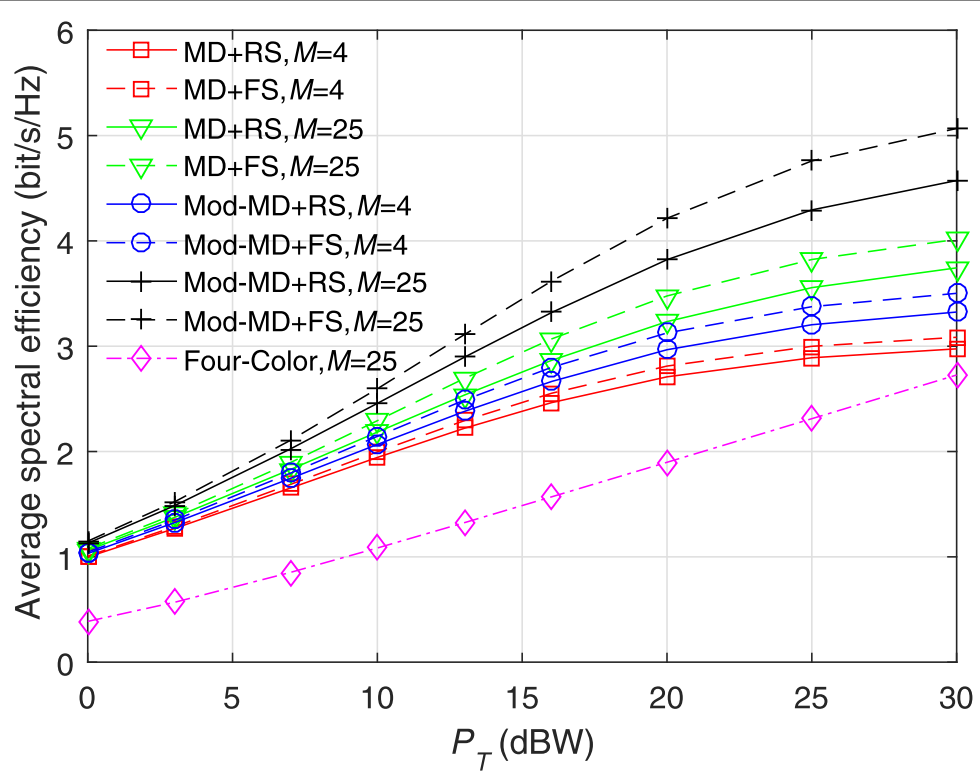

(a)

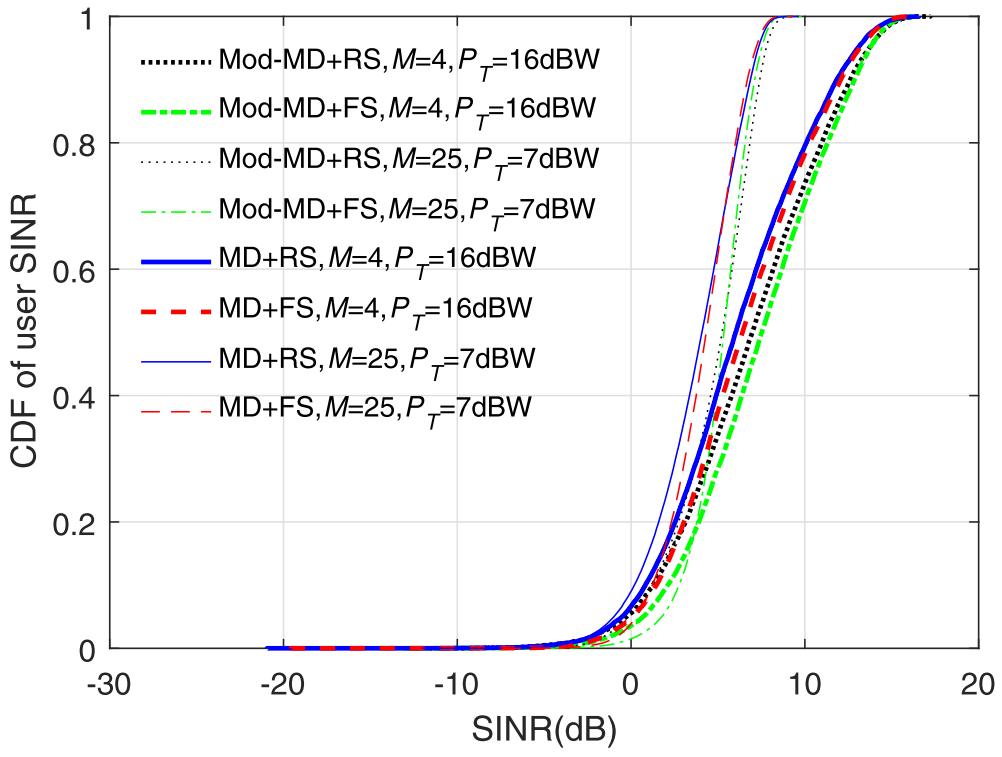

(b)

Fig. 4 Spectral efficiency verse $P_{T}, R=2$. a Average spectral efficiency. $\mathbf{b}$ CDF of user SINR with specific parameters 
performance than those with RS. With the rise in $R$, the performance difference between $\mathrm{MD}+\mathrm{FS}$ and $\mathrm{MD}+\mathrm{RS}$ becomes small. This is due to the fact that MD mainly concerns the similarity of the group members and the increased interference caused by the growth in $R$ weakens the effectiveness of FS. In contrast to MD, Mod-MD takes account of the interference among the beams during the scheduling. This peculiarity of Mod-MD results in a consistent performance increase for Mod-MD+FS by comparison with Mod-MD+RS.

Figures 4 and 5 present the spectral efficiency verse $P_{T}$ under different scheduling schemes with different values of $M$ and $R$. The cumulative distribution function (CDF)

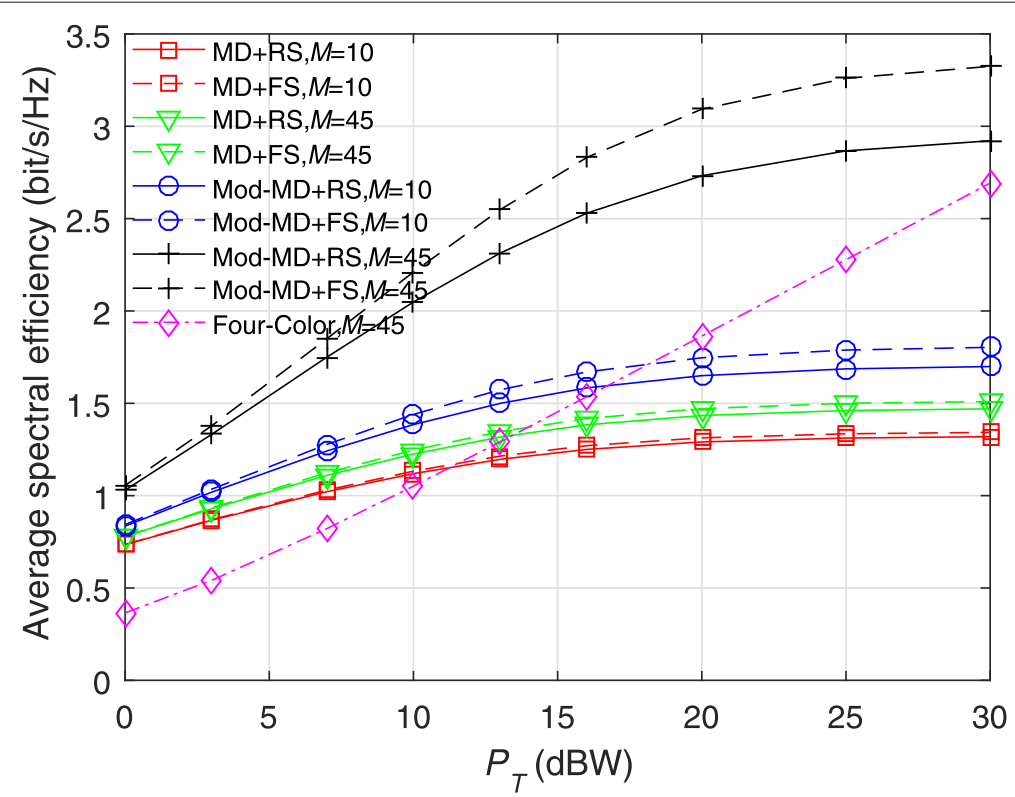

(a)

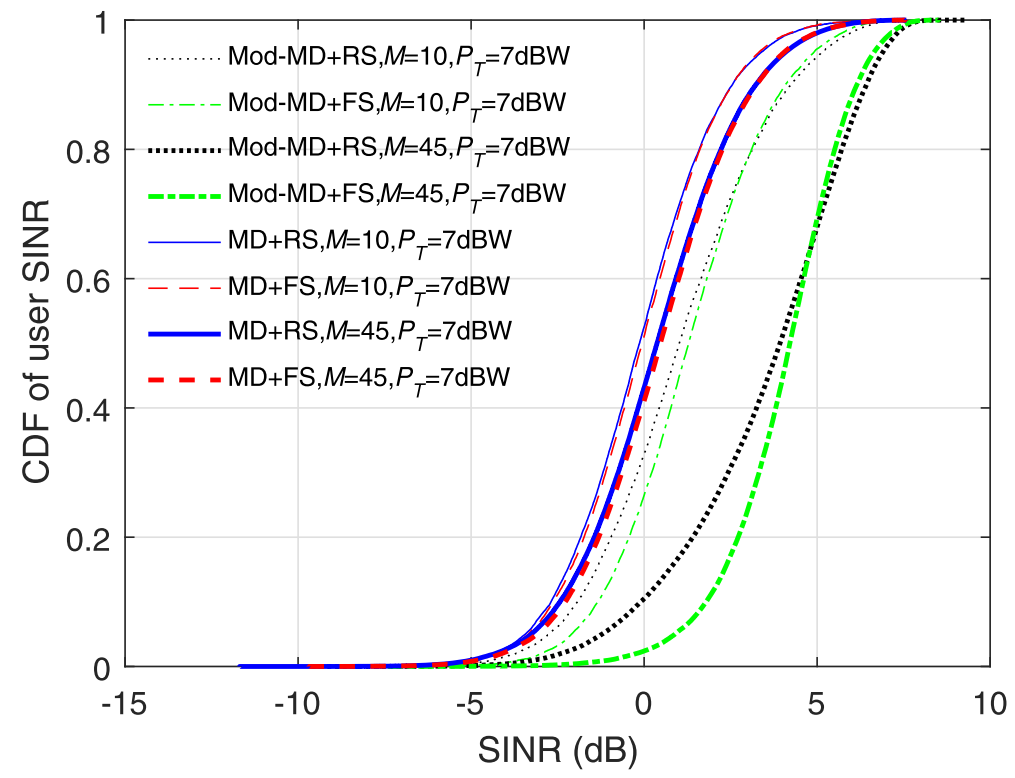

(b)

Fig. 5 Spectral efficiency verse $P_{T}, R=5$. a Average spectral efficiency. $\mathbf{b}$ CDF of user SINR with specific parameters, $P_{T}=7 d B W$ 
curves of user SINR with specific parameters are depicted as supplements in Figs. 4 and 5. User SINR is the SINR corresponding to the actual transmission rate of the user. Four-color refers to the multicast transmission in the system with four-color frequency reuse where $P_{T}$ is uniformly distributed among the seven beams. For different values of $P_{T}$, especially at low $P_{T}$, the average spectral efficiency under the four-color frequency reuse scenario is inferior to that under the full frequency reuse scenario with user scheduling schemes, owing largely to the reduced available bandwidth in each beam. It is also seen that, with the rise in $P_{T}$, the spectral efficiency increases under the four scheduling schemes gradually decline, while the spectral efficiency under the four-color

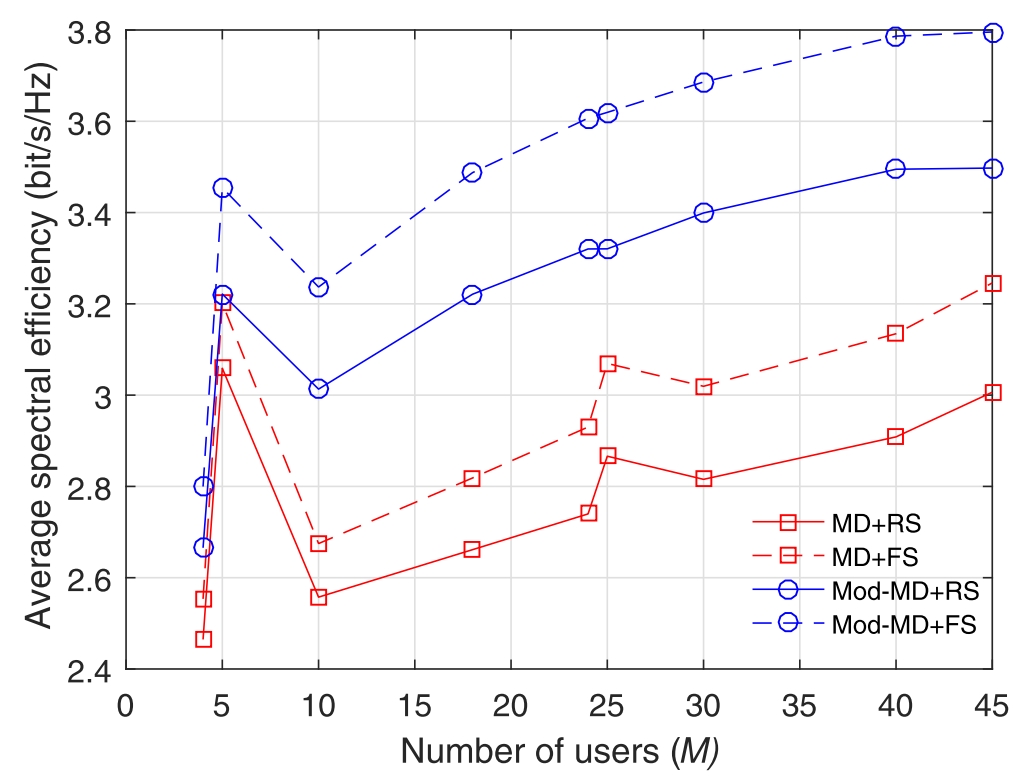

(a)

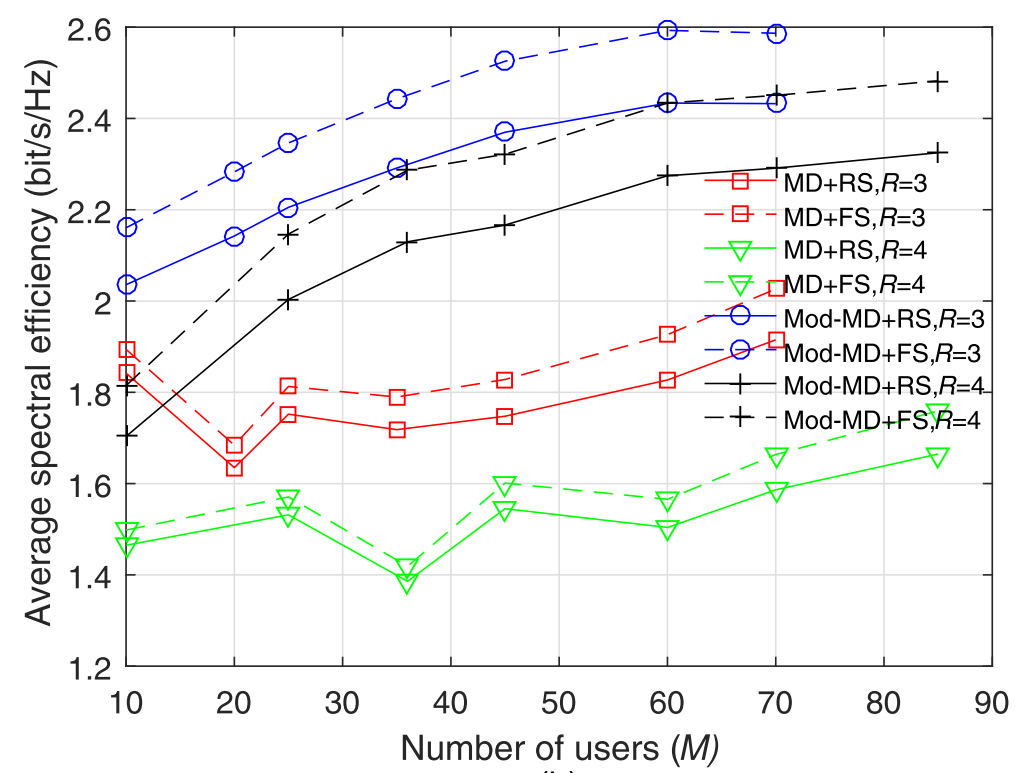

(b)

Fig. 6 Average spectral efficiency verse $M$. a $R=2, P_{T}=13 d B W$. b $P_{T}=7 d B W$ 
scenario shows an almost linear growth. The results indicate that the system with fourcolor frequency reuse is power-limited, and system with full-frequency reuse scenario is interference-limited.

In Fig. 6, the average spectral efficiency verse $M$ under different scheduling schemes is plotted. It is seen that Mod-MD outperforms MD. The advantage of Mod-MD lies in the consideration of interference-limited characteristics of the system. In most cases, the average spectral efficiency of different scheduling schemes increases with the growth in $M$. This stems from the larger multiuser diversity gain caused by the increase in $M$. However, exceptions exist with regard to specific values of $M$, such as $M=4,5$ in Fig. 6a, and $M=25,36$ in Fig. $6 \mathrm{~b}$. This is due to the fact that $M$ is a non-integer multiple of $R$, i.e., the size of one frame group is smaller than $R$. Since the average spectral efficiency may decrease with the increase in $R$, the users in the frame group with the smaller size may have higher spectral efficiency than the others. However, the exceptions are nonsignificant for Mod-MD. A possible explanation of this might be that the performance enhancement caused by Mod-MD covers the effect caused by the frame group with the smaller size.

In Fig. 7, we provide the average spectral efficiency verse $K$ under different scheduling schemes. For each set of $K$ and $R$, it satisfies that each frame group has the same number of users. It is observed that the average spectral efficiency increases with the growth in $K$ under all the four scheduling schemes. Since the effect caused by the unequal group sizes in each beam is eliminated, the simulation results indicate the multiuser diversity gain caused by the growth in $M$ for user scheduling.

The above simulation results reveal that Mod-MD+FS stands out from the other scheduling schemes in terms of the average spectral efficiency. Moreover, Mod-MD and FS can be separately employed to increase the average spectral efficiency of the system, i.e., Mod-MD+RS and MD+FS both outperform MD+RS.

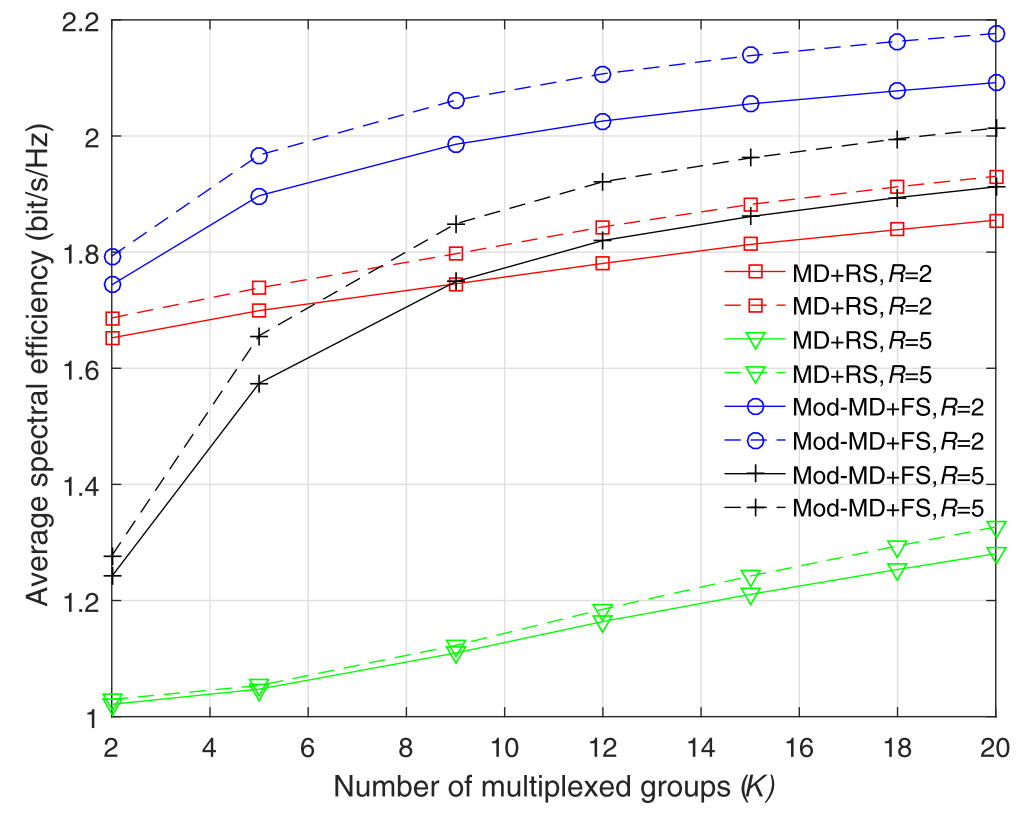

Fig. 7 Average spectral efficiency verse $K, P_{T}=7 \mathrm{dBW}$ 


\subsection{Jain's fairness index}

Jain's fairness index is utilized as the indicator to evaluate the fairness among users. For a series of variables $x_{1}, x_{2}, \ldots, x_{n}$, Jain's fairness index is defined as

$$
\mathcal{J}\left(x_{1}, x_{2}, \ldots, x_{n}\right)=\frac{\left(\sum_{i=1}^{n} x_{i}\right)^{2}}{n \sum_{i=1}^{n} x_{i}^{2}} .
$$

In this simulation, each Jain's fairness index is calculated based on (20) with the spectral efficiency of the $N M$ users.

Figures 8 and 9 show the Jain's fairness index verse $P_{T}$ under different scheduling schemes, including the average Jain's fairness index and the CDF of Jain's fairness index

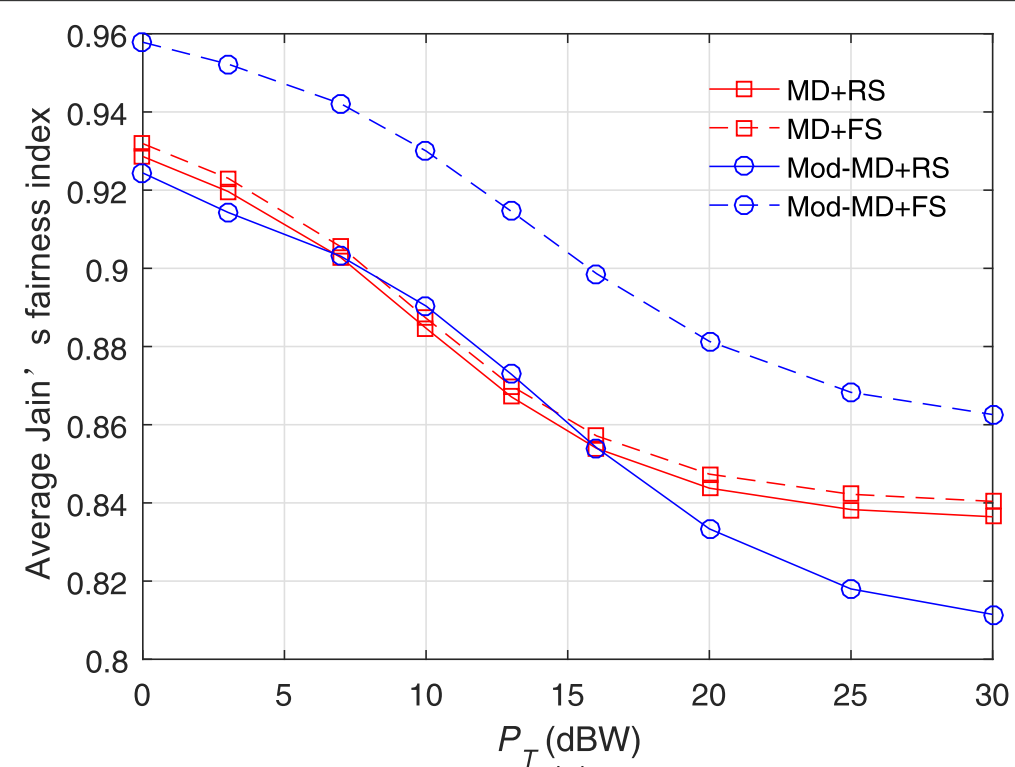

(a)

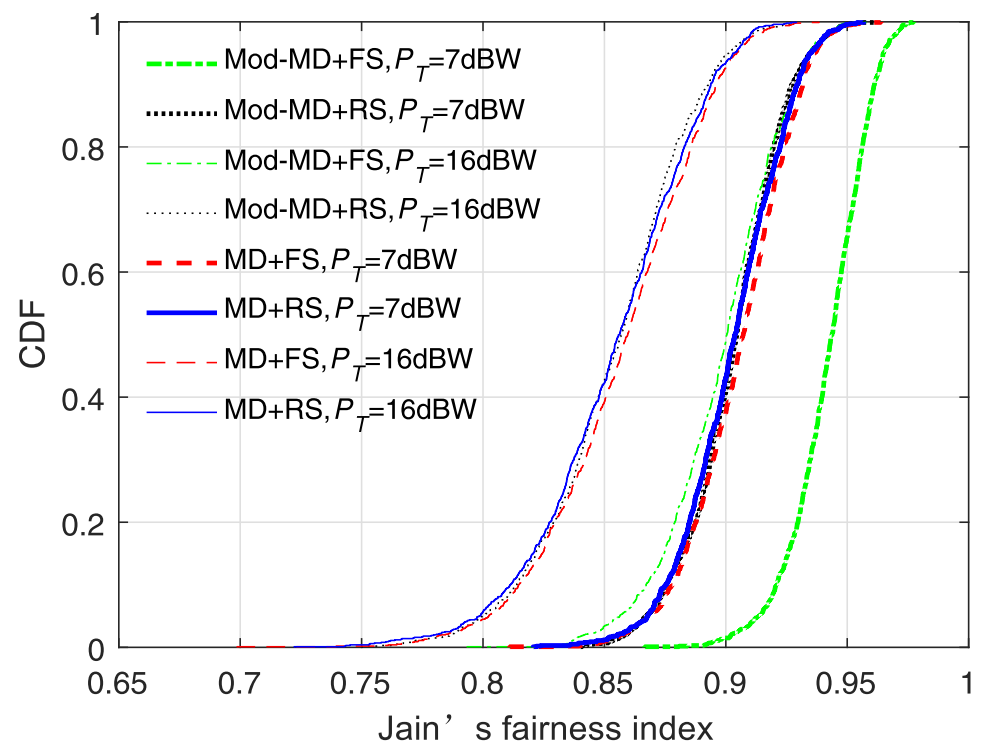

(b)

Fig. 8 Jain's fairness index verse $P_{T}, R=5, M=25$. a Average Jain's fairness index. $\mathbf{b}$ CDF of Jain's fairness index with specific parameters 


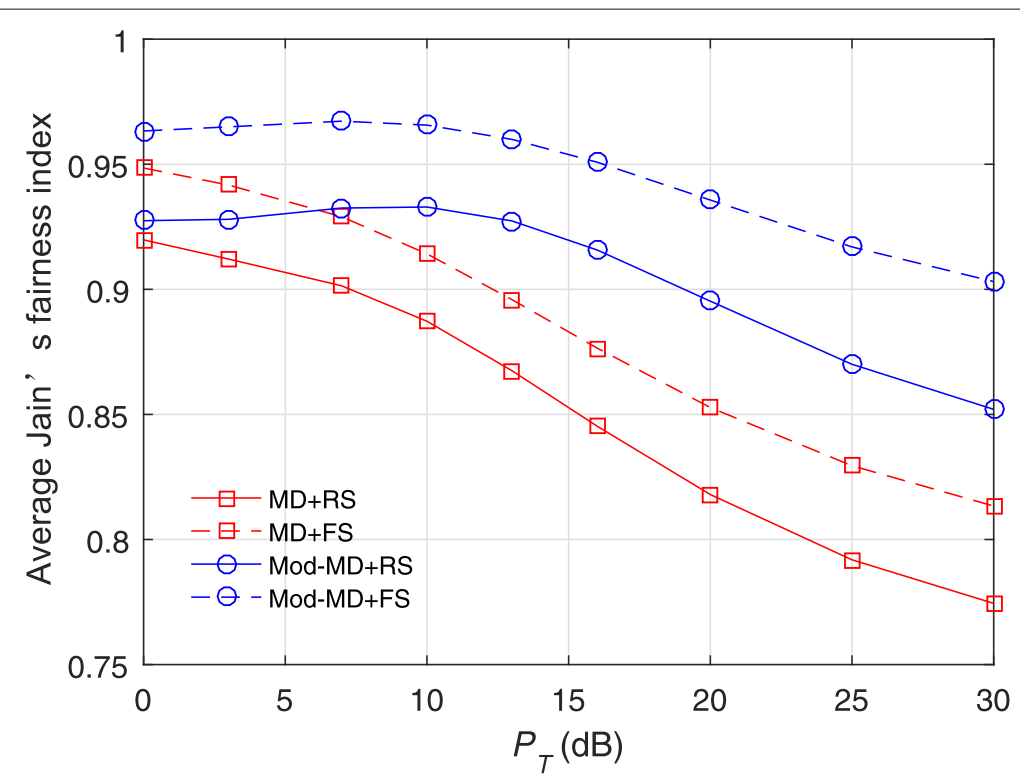

(a)

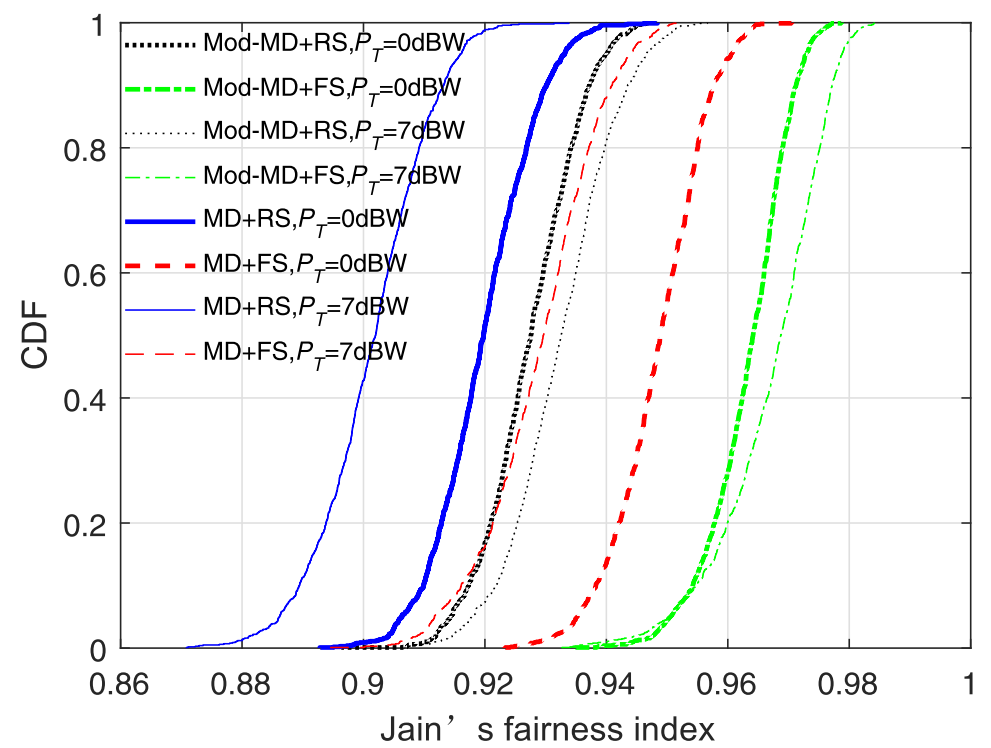

(b)

Fig. 9 Jain's fairness index verse $P_{T}, R=3, M=70$. a Average Jain's fairness index. $\mathbf{b}$ CDF of Jain's fairness index with specific parameters

with respect to specific values of $P_{T}$. It is seen that the schemes with FS perform better than those with RS. This indicates the superiority of FS. With the increase in $P_{T}$, for all the scheduling schemes, the average Jain's fairness index shows a downward trend. This is expected as the increased spectral efficiency caused by the rise in $P_{T}$ leads to larger data rate differences among the users. As shown in Fig. 10 , the curves with different values of $P_{T}$ imply that the user SINR range becomes larger with the increase in $P_{T}$. For large values of $P_{T}$, not the noise but the inter-beam interference has great effects on user data rates and the interference differences may be considerable. This may explain the downtrend of the average Jain's fairness index. It is also observed that the increase in user SINR 


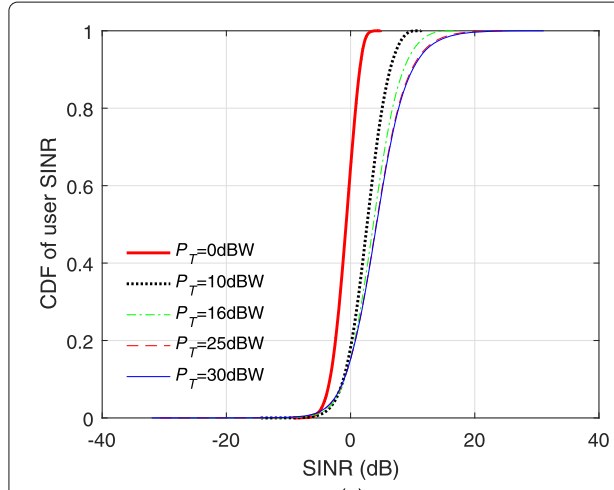

(a)

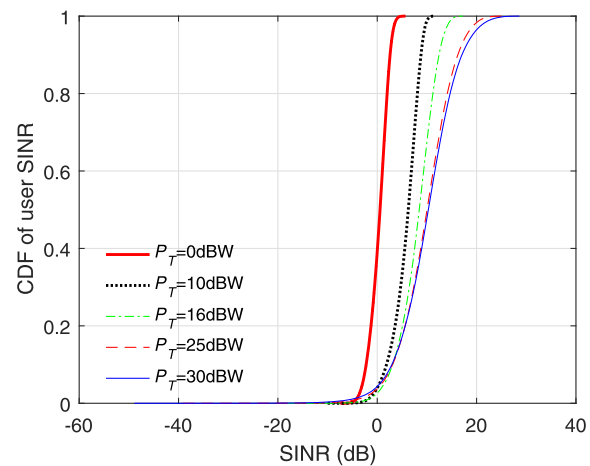

(c)

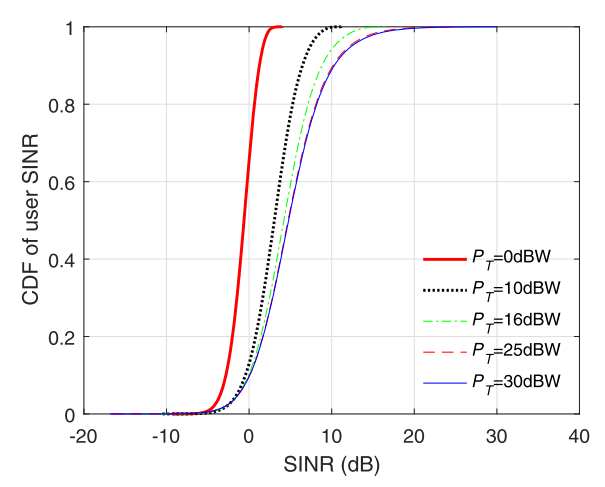

(b)

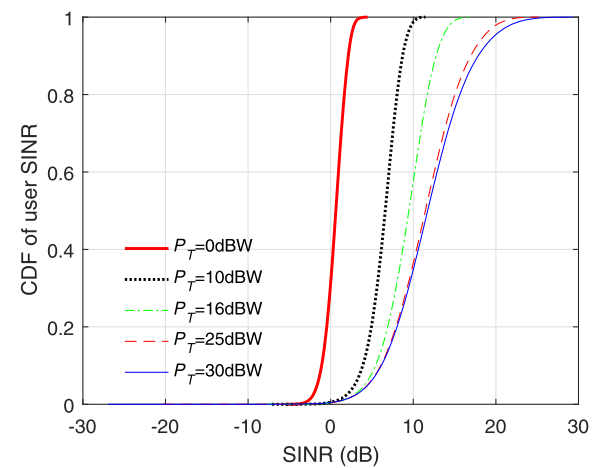

(d)

Fig. 10 CDF of user SINR, $R=4, M=70$. a MD+RS. b MD+FS. c Mod-MD+RS. d Mod-MD+FS

declines with the increase in $P_{T}$, owing largely to the interference-limited characteristics of the system.

In Figs. 11 and 12, we plot the Jain's fairness index verse $R$ under different scheduling schemes with $M=90,120$. It is seen that Mod-MD+FS is superior to the others and, in most cases, FS can bring the enhancement of fairness by comparison with RS. Precisely, compared with RS, FS can bring almost consistent fairness enhancement for Mod-MD+FS, while the fairness enhancement declines with the increase in $R$ for MD+FS. The performance difference stems from the different characteristics of MD and ModMD. It is also seen that the Jain's fairness index with MD shows an increasing trend. This is expected due to the increase in $R$ with fixed $M$. The slight reduction of fairness in ModMD with the increase in $R$ is due to the ignorance of some channel coefficients. However, Mod-MD still outperforms MD in most cases.

Figures 13 and 14 depict the average Jain's fairness index verse $K$ and $M$, respectively. In the simulation of Fig. 13, each $M$ is an integral multiple of the corresponding $K$. It is observed that the average Jain's fairness index increases with the rise in $K(M)$, especially for the schemes with Mod-MD. Since the space of the scheduling results grows exponentially with the increase in $K$, the probability that RS can achieve favorable scheduling results becomes small. The simulation results show that FS can provide performance gain by comparison with RS. Additionally, Fig. 13 indicates the increased multiuser diversity gain with respect to the growth in $M$. When $M$ is a non-integral multiple of $R$, the frame groups with smaller sizes may impact the fairness among users. For example, in Fig. 14, when $R=3$, the average Jain's fairness index with $M=20$ is higher than that with $M=25$ 


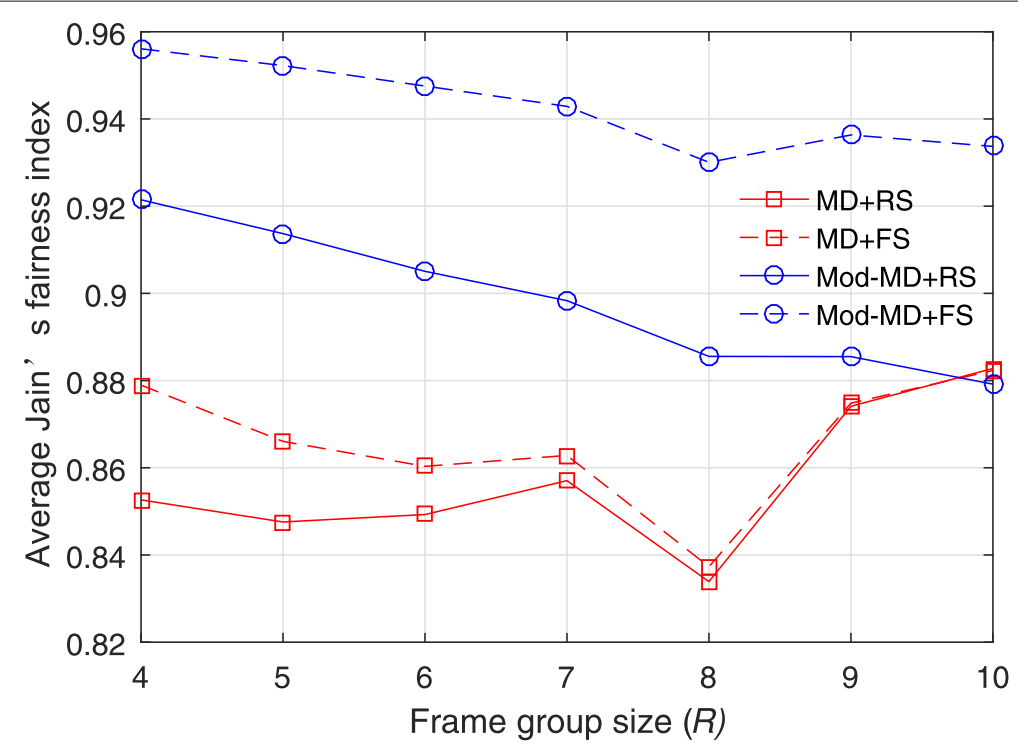

(a)



(b)

Fig. 11 Jain's fairness index verse $R, M=90, P_{T}=13 d B W$. a Average Jain's fairness index. b CDF of Jain's fairness index with specific parameters

under the schemes with MD. The users in these frame groups with smaller sizes may have higher data rates than the others, which leads to unfairness among the users. However, the effect caused by the frame groups with smaller sizes is small for Mod-MD. This is because Mod-MD can achieve favorable performance for all the users.

Above all, the schemes with FS can achieve better fairness performance than those with RS. In most cases, Mod-MD outperforms MD due to the fact that it emphasizes the interference among the beams. This fact can bring about apparent fairness enhancement. Moreover, the joint use of Mod-MD and FS can always achieve better performance than the other scheduling schemes. 


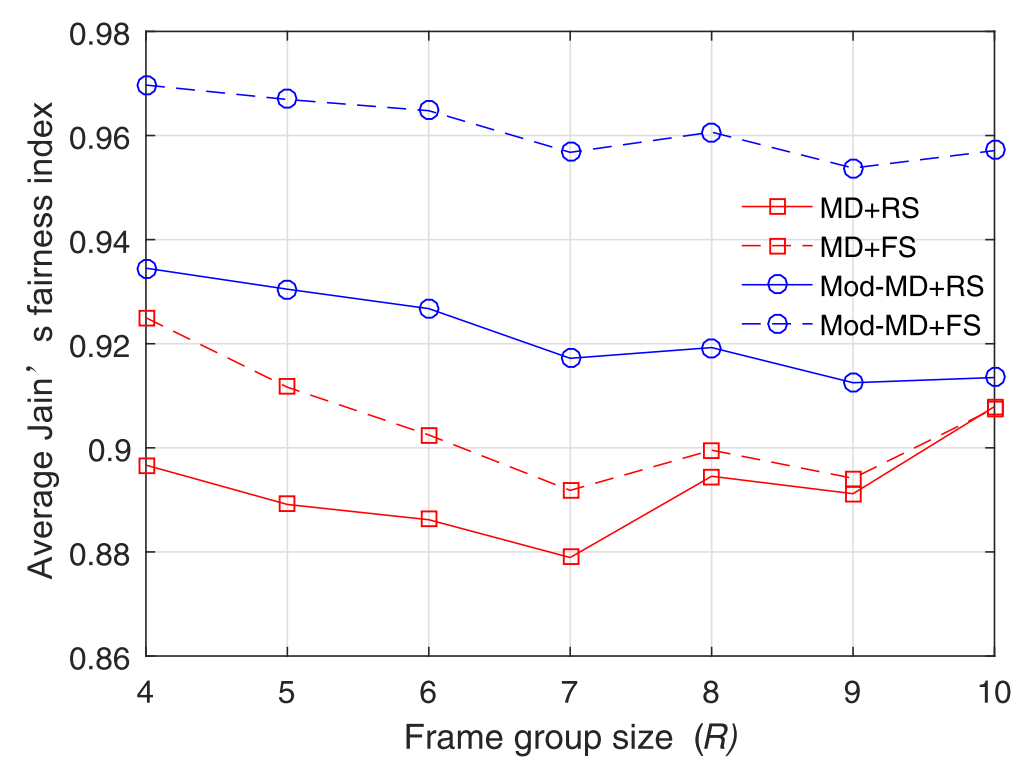

(a)

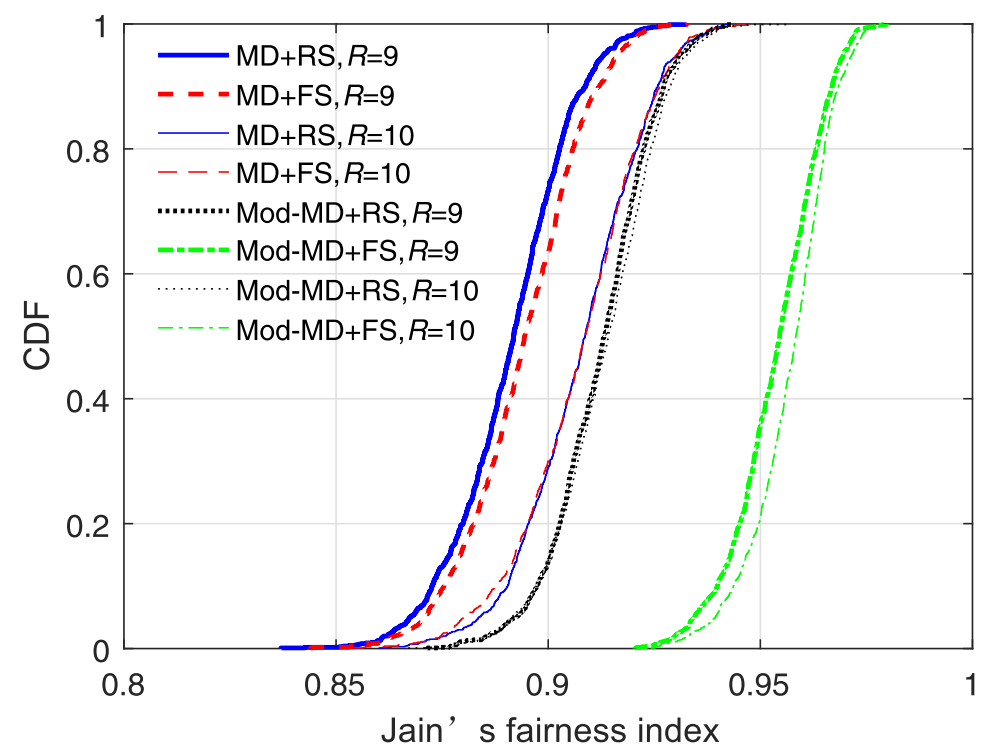

(b)

Fig. 12 Jain's fairness index verse $R, M=120, P_{T}=7 d B W$. a Average Jain's fairness index. $\mathbf{b}$ CDF of Jain's fairness index with specific parameters

\section{Conclusion}

This paper addressed the user scheduling problem for the multicast transmission in HTS systems with full frequency reuse. The scheduling problem has been divided into intrabeam and inter-beam scheduling. For the intra-beam scheduling problem, a fixed-size user grouping algorithm has been proposed. For the inter-beam scheduling problem, first, the concept of equivalent CSIs has been introduced to simplify the scheduling problem by making each scheduled unit correspond to only one CSI and two forms of equivalent CSIs have been proposed. Second, according to equivalent CSIs, a scheduling algorithm aiming 


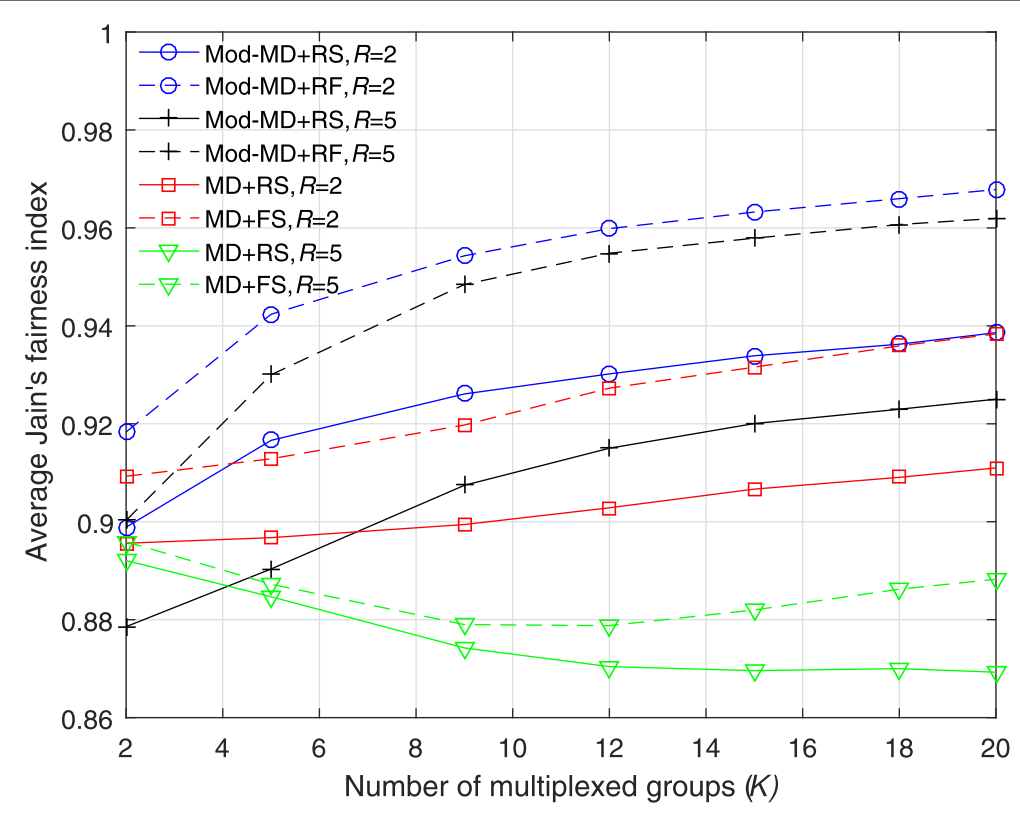

Fig. 13 Average Jain's fairness index verse $K_{,} P_{T}=10 \mathrm{dBW}$

at improving the fairness has been proposed. The simulation results show that, in most cases, either the proposed intra-beam or inter-beam scheduling algorithm can improve the performance with respect to system spectral efficiency and user fairness. Moreover, the joint use of the two proposed algorithms can achieve a huge performance increase in the system.

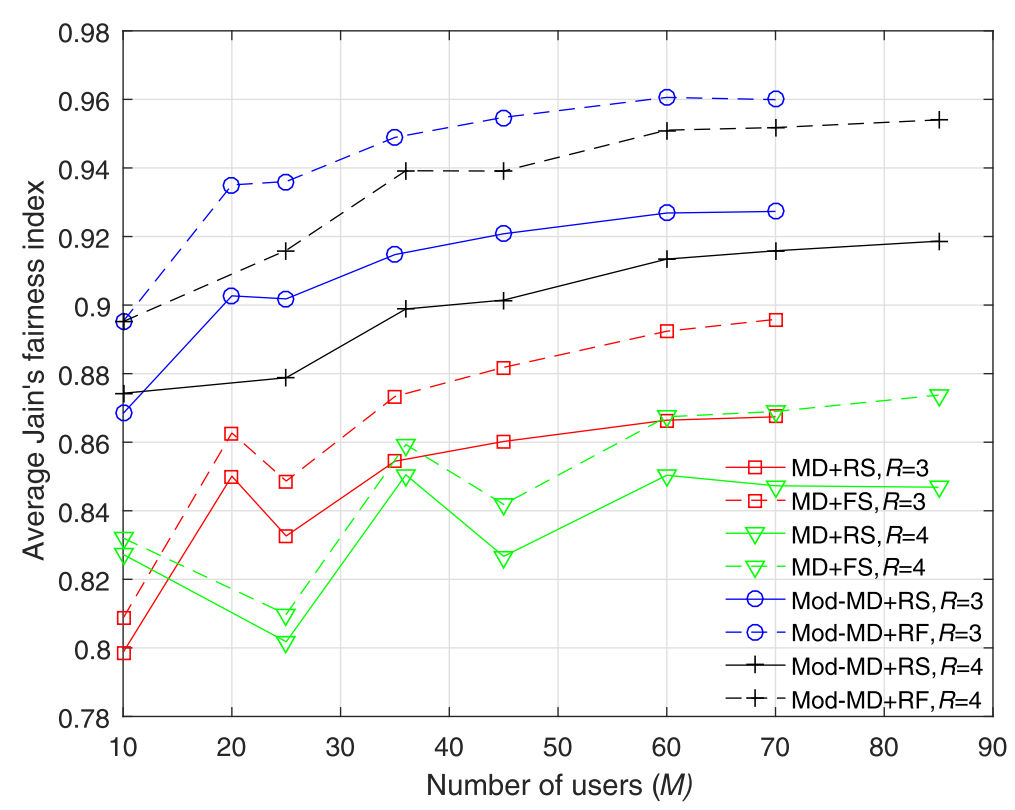

Fig. 14 Average Jain's fairness index verse $M, P_{T}=13 d B W$ 


\section{Abbreviations}

HTS: High throughput satellite; MIMO: Multiple Input Multiple Output; CSI: Channel state information; MMSE: Minimum mean squared error; DVB-S2: Digital video broadcasting-satellite 2; LDPC: Low density parity check; SFB: Single feed per beam; RF: Radio frequency; SINR: Signal to interference plus noise ratio; NoIP: Norm of the inner product; FS: fairness scheduling; MD: MaxDist; Mod-MD: Mod-MaxDist; RS: Random scheduling; CDF: Cumulative distribution function

\section{Acknowledgements}

This work was supported by the National Natural Science Foundation of China (No.61671183, 61771163).

\section{Authors' contributions}

SZ and QG put forward the idea of this paper. SZ finished the design of the study and the algorithms. YW and MJ contributed to the experimental work and the data analysis. SZ and MJ drafted the manuscript. The authors read and approved the manuscript.

\section{Funding}

National Natural Science Foundation of China under grant numbers 61671183 and 61771163.

\section{Availability of data and materials}

Data sharing is not applicable to this article as no datasets were generated or analyzed during the current study.

\section{Competing interests}

The authors declare that they have no competing interests.

Received: 14 February 2020 Accepted: 3 June 2020

Published online: 26 June 2020

\section{References}

1. D. H. Werner, S. Ganguly, An overview of fractal antenna engineering research. IEEE Antennas Propag. Mag. 45(1), 38-57 (2003)

2. E. Guariglia, Harmonic sierpinski gasket and applications. Entropy. 20(9), 1-17 (2018)

3. K. C. Hwang, A modified Sierpinski fractal antenna for multiband application. IEEE Antennas Wirel. Propag. Lett. 6 357-360 (2007)

4. E. Guariglia, Entropy and fractal antennas. Entropy. 18(3), 1-12 (2016)

5. R. G. Hohlfeld, N. Cohen, Self-similarity and the geometric requirements for frequency independence in antennae. Fractals. 07(01), 79-84 (1999). https://doi.org/10.1142/S0218348X99000098

6. E. Guariglia, in 2017 2nd International Multidisciplinary Conference on Computer and Energy Science (SpliTech), Spectral analysis of the weierstrass-mandelbrot function (IEEE, Split, Croatia, 2017), pp. 1-6

7. E. Martinez-de-Rioja, D. Martinez-de-Rioja, J. A. Encinar, A. Pino, B. Gonzalez-Valdes, Y. Rodriguez-Vaqueiro, M. Arias, G. Toso, Advanced multibeam antenna configurations based on reflectarrays: providing multispot coverage with a smaller number of apertures for satellite communications in the K and Ka bands. IEEE Antennas Propag. Mag. 61(5), 77-86 (2019). https://doi.org/10.1109/MAP.2019.2932311

8. H. Fenech, A. Tomatis, S. Amos, V. Soumpholphakdy, J. L. Serrano Merino, Eutelsat HTS systems. Int. J. Satell. Commun. Netw. 34(4), 503-521 (2016). https://doi.org/10.1002/sat.1171

9. Y. Guan, F. Geng, J. H. Saleh, Review of high throughput satellites: market disruptions, affordability-throughput map, and the cost per bit/second decision tree. IEEE Aerosp. Electron. Syst. Mag. 34(5), 64-80 (2019). https://doi.org/10. 1109/MAES.2019.2916506

10. R. De Gaudenzi, P. Angeletti, D. Petrolati, E. Re, Future technologies for very high throughput satellite systems. Int. J. Satell. Commun. Netw. 38, 1-21 (2019). https://doi.org/10.1002/sat.1327

11. L. Cottatellucci, M. Debbah, G. Gallinaro, R. Mueller, M. Neri, R. Rinaldo, in Proc. AlAA International Communications Satellite Systems Conference (ICSSC2006), Interference mitigation techniques for broadband satellite systems (American Institute of Aeronautics and Astronautics, San Diego, USA, 2006), pp. 1-13. https://doi.org/10.2514/6. 2006-5348

12. D. Christopoulos, P. D. Arapoglou, S. Chatzinotas, in Proc. AlAA International Communications Satellite Systems Conference (ICSSC2003), Linear precoding in multibeam satcoms: practical constraints (American Institute of Aeronautics and Astronautics, Florence, Italy, 2013), pp. 1-9. https://doi.org/10.2514/6.2013-5716

13. J. Arnau, B. Devillers, C. Mosquera, A. Pérez-Neira, Performance study of multiuser interference mitigation schemes for hybrid broadband multibeam satellite architectures. EURASIP J. Wirel. Commun. Netw. 2012(1), 132 (2012). https://doi.org/10.1186/1687-1499-2012-132

14. Z. Gan, S. Chatzinotas, B. Ottersten, Generic optimization of linear precoding in multibeam satellite systems. IEEE Trans. Wirel. Commun. 11(6), 2308-2320 (2012). https://doi.org/10.1109/twc.2012.040412.111629

15. Digital Video Broadcasting (DVB); Implementation guidelines for the second generation system for Broadcasting, Interactive Services, News Gathering and other broadband satellite applications. DVB Document A171-2, 149-178 (2015)

16. V. Joroughi, M. A. Vazquez, A. I. Perez-Neira, Generalized multicast multibeam precoding for satellite communications. IEEE Trans. Wirel. Commun. 16(2), 952-966 (2017). https://doi.org/10.1109/TWC.2016.2635139

17. H. Fenech, A. Tomatis, D. Serrano, E. Lance, M. Kalama, Spacecraft antenna requirements as perceived by an operator. IEEE Antennas Propag. Mag. 53(5), 256-266 (2011). https://doi.org/10.1109/MAP.2011.6138491

18. D. Christopoulos, S. Chatzinotas, B. Ottersten, Multicast multigroup precoding and user scheduling for frame-based satellite communications. IEEE Trans. Wirel. Commun. 14(9), 4695-4707 (2015). https://doi.org/10.1109/twc.2015. 2424961 
19. A. Guidotti, A. Vanelli-Coralli, Clustering strategies for multicast precoding in multibeam satellite systems. Int. J. Satell. Commun. Netw. 38(2), 85-104 (2020). https://doi.org/10.1002/sat.1312

20. E. Lagunas, S. Andrenacci, S. Chatzinotas, B. Ottersten, in Proc. 9th Advanced Satellite Multimedia Systems Conference and the 15th Signal Processing for Space Communications Workshop (ASMS/SPSC2018), Cross-layer forward packet scheduling for emerging precoded broadband multibeam satellite system (IEEE, Berlin, Germany, 2018), pp. 1-8. https://doi.org/10.1109/ASMS-SPSC.2018.8510717

21. Digital Video Broadcasting (DVB); Second generation framing structure, channel coding and modulation systems for Broadcasting, Interactive Services,News Gathering and other broadband satellite applications. ETSI EN 302 307-1, 13-15 (2014)

22. M. A. Vazquez, M. R. B. Shankar, C. I. Kourogiorgas, P. Arapoglou, V. Icolari, S. Chatzinotas, A. D. Panagopoulos, A. I. Pérez-Neira, Precoding, scheduling, and link adaptation in mobile interactive multibeam satellite systems. IEEE J. Sel. Areas Commun. 36(5), 971-980 (2018). https://doi.org/10.1109/JSAC.2018.2832778

23. Y. Taesang, A. Goldsmith, On the optimality of multiantenna broadcast scheduling using zero-forcing beamforming. IEEE J. Sel. Areas Commun. 24(3), 528-541 (2006). https://doi.org/10.1109/JSAC.2005.862421

24. A. Guidotti, A. Vanelli-Coralli, in Proc. 9th Advanced Satellite Multimedia Systems Conference and the 15th Signal Processing for Space Communications Workshop (ASMS/SPSC2018), Geographical scheduling for multicast precoding in multi-beam satellite systems (IEEE, Berlin, Germany, 2018), pp. 1-8

25. D. Christopoulos, S. Chatzinotas, B. Ottersten, in Cooperative and Cognitive Satellite Systems. ed. by S. Chatzinotas, B. Ottersten, and R. De Gaudenzi, User scheduling in cooperative satellite systems (Academic Press, UK, 2015), pp. 217-244. https://doi.org/10.1016/B978-0-12-799948-7.00007-4 Chap. 7

26. A. I. Perez-Neira, M. A. Vazquez, M. R. B. Shankar, S. Maleki, S. Chatzinotas, Signal processing for high-throughput satellites: challenges in new interference-limited scenarios. IEEE Signal Proc. Mag. 36(4), 112-131 (2019). https://doi. org/10.1109/MSP.2019.2894391

27. M. A. Vazquez, A. Perez-Neira, D. Christopoulos, S. Chatzinotas, B. Ottersten, P. Arapoglou, A. Ginesi, G. Tarocco, Precoding in multibeam satellite communications: present and future challenges. IEEE Wirel. Commun. 23(6), 88-95 (2016). https://doi.org/10.1109/mwc.2016.1500047wc

28. A. Morello, V. Mignone, DVB-S2X: extending DVB-S2 flexibility for core markets and new applications. Int. J. Satell. Commun. Netw. 34(3), 327-336 (2016). https://doi.org/10.1002/sat.1157

29. D. Christopoulos, S. Chatzinotas, B. Ottersten, Weighted fair multicast multigroup beamforming under per-antenna power constraints. IEEE Trans. Signal Process. 62(19), 5132-5142 (2014). https://doi.org/10.1109/tsp.2014.2345340

30. D. Christopoulos, S. Chatzinotas, B. Ottersten, in Proc. IEEE International Conference on Communications (ICC2014), Multicast multigroup beamforming under per-antenna power constraints (IEEE, Sydney, Australia, 2014), pp. 4704-4710. https://doi.org/10.1109//CC.2014.6884064

31. G. Taricco, in Proc. European Wireless Conference'2014, Linear precoding methods for multi-beam broadband satellite systems (VDE, Barcelona, Spain, 2014), pp. 1-6

32. J. Han, M. Kamber, J. Pei, in Data Mining (Third Edition). ed. by J. Han, M. Kamber, and J. Pei, Getting to know your data (Morgan Kaufmann, Boston, 2012), pp. 39-82. https://doi.org/10.1016/B978-0-12-381479-1.00002-2 Chap. 2.

33. N. D. Sidiropoulos, T. N. Davidson, Z. Q. Luo, Transmit beamforming for physical-layer multicasting. IEEE Trans. Signal Process. 54(6), 2239-2251 (2006). https://doi.org/10.1109/Tsp.2006.872578

34. A. I. Aravanis, M. R. B. Shankar, P. Arapoglou, G. Danoy, P. G. Cottis, B. Ottersten, Power allocation in multibeam satellite systems: a two-stage multi-objective optimization. IEEE Trans. Wirel. Commun. 14(6), 3171-3182 (2015). https://doi.org/10.1109/twc.2015.2402682

\section{Publisher's Note}

Springer Nature remains neutral with regard to jurisdictional claims in published maps and institutional affiliations.

\section{Submit your manuscript to a SpringerOpen ${ }^{\circ}$ journal and benefit from:}

$\rightarrow$ Convenient online submission

- Rigorous peer review

- Open access: articles freely available online

- High visibility within the field

- Retaining the copyright to your article

Submit your next manuscript at $\gg$ springeropen.com 